\title{
Vector and tensor analyzing powers in deuteron-proton breakup at $130 \mathrm{MeV}$
}

\author{
E. Stephan, ${ }^{1,}{ }^{*}$ St. Kistryn, ${ }^{2}$ R. Sworst, ${ }^{2}$ A. Biegun, ${ }^{1}$ K. Bodek, ${ }^{2}$ I. Ciepał,${ }^{2}$ A. Deltuva, ${ }^{3}$ E. Epelbaum, ${ }^{4}$ A. C. Fonseca, ${ }^{5}$ \\ J. Golak, ${ }^{2}$ N. Kalantar-Nayestanaki, ${ }^{6}$ H. Kamada,${ }^{7}$ M. Kiš ${ }^{6}{ }^{6}$ B. Kłos, ${ }^{1}$ A. Kozela, ${ }^{8}$ M. Mahjour-Shafiei,${ }^{6, \dagger}$ A. Micherdzińska, ${ }^{1, \dagger}$ \\ A. Nogga, ${ }^{9}$ R. Skibiński, ${ }^{2}$ H. Witała, ${ }^{2}$ A. Wrońska, ${ }^{2}$ J. Zejma, ${ }^{2}$ and W. Zipper ${ }^{1}$ \\ ${ }^{1}$ Institute of Physics, University of Silesia, PL-40007 Katowice, Poland \\ ${ }^{2}$ Institute of Physics, Jagiellonian University, PL-30059 Kraków, Poland \\ ${ }^{3}$ Centro de Física Nuclear da Universidade de Lisboa, P-1649-003 Lisboa, Portugal \\ ${ }^{4}$ Institute für Theoretische Physik II, Ruhr-Universität Bochum, D-44780 Bochum, Germany \\ ${ }^{5}$ Centro de Física Nuclear, Universidade de Lisboa, P-1649-003 Lisboa, Portugal \\ ${ }^{6}$ Kernfysisch Versneller Instituut, University of Groningen, NL-9747 AA Groningen, The Netherlands \\ ${ }^{7}$ Department of Physics, Kyushu Institute of Technology, Kitakyushu 804-8550, Japan \\ ${ }^{8}$ Institute of Nuclear Physics, Polish Academy of Sciences, PL-31342 Kraków, Poland \\ ${ }^{9}$ Forschungszentrum Jülich, Institut für Kernphysik, Institute for Advanced Simulations, and \\ Jülich Center for Hadron Physics, D-52425 Jülich, Germany
}

(Received 19 April 2010; revised manuscript received 8 June 2010; published 23 July 2010)

\begin{abstract}
High-precision data for vector and tensor analyzing powers for the ${ }^{1} \mathrm{H}(\vec{d}, p p) n$ reaction at a 130-MeV deuteron beam energy have been measured over a large part of the phase space. Theoretical predictions based on various approaches to describe the three nucleon $(3 N)$ system reproduce very well the vector analyzing power data and no three-nucleon force effect is observed for these observables. Tensor analyzing powers are also very well reproduced by calculations in almost the whole studied region, but locally certain discrepancies are observed. For $A_{x y}$ such discrepancies usually appear, or are enhanced, when model $3 N$ forces, TM99 or Urbana, are included. Problems of all theoretical approaches with describing $A_{x x}$ and $A_{y y}$ are limited to very small kinematical regions, usually characterized by the lowest energy of the relative motion of the two protons.
\end{abstract}

DOI: 10.1103/PhysRevC.82.014003

PACS number(s): 21.30.-x, 21.45.Ff, 24.70.+s, 25.10.+s

\section{INTRODUCTION}

The successes of meson-exchange theories in the description of two-nucleon observables have been followed in the past few years by intensive studies of three nucleon $(3 N)$ systems. Numerically exact predictions and precise experimental data for such systems prepared the basis for tracing inconsistencies between a theoretical description limited to pairwise $N N$ interactions only and the measured data, which can be assigned to additional dynamics related to the presence of the third nucleon, the so-called three-nucleon force (3NF). A number of models and approaches to introduce 3 NFs into calculations have emerged, seeking, for an even larger data base for their tests and developments.

The importance of $3 \mathrm{NF}$ contributions for the proper description of systems of more than two nucleons was first established in few-nucleon bound states [1-4]. When exact calculations including $3 \mathrm{NF}$ became available for observables in the nucleon-deuteron elastic scattering, the present models of $3 \mathrm{NF}$ turned out to be an efficient remedy for a longpersisting problem of describing cross section for this process [5-7]. Though at energies above $100 \mathrm{MeV}$ some problems with describing the data have been observed (cf. Ref. [8]

\footnotetext{
*elzbieta.stephan@us.edu.pl

†Present address: Department of Physics, University of Teheran, Iran.

${ }^{\ddagger}$ Present address: The George Washington University, Washington, DC 20052, USA.
}

and references therein), the improvement of the description of the cross-section data due to the inclusion of $3 \mathrm{NFs}$ is still considerable. On the other hand, precise experimental data demonstrate both the successes and difficulties of the current models in describing analyzing powers, spin transfer and spin correlation coefficients [9-17]. This indicates problems with the spin part of current models of the $3 \mathrm{~N}$ interaction.

In addition to the bound states and elastic scattering, the $3 \mathrm{~N}$ system can be studied via the deuteron-nucleon breakup reaction, which is characterized by the very rich kinematics of the final state. The complexity of this process poses a challenge for both theoretical calculations and experimental investigations but, on the other hand, it opens a way to study kinematical dependencies of the $3 \mathrm{NF}$ effects, possibly giving insight into the underlying dynamics. Due to the advances in experimental techniques, like the availability of highly polarized beams in a wide range of energies, construction of position-sensitive, high-acceptance detectors, and the development of efficient data-acquisition systems, data of high accuracy and covering large kinematical regions can be collected. The database for the breakup reaction in the region of intermediate energies has grown, which is very encouraging, since until recently cross sections and analyzing powers were only measured at $65 \mathrm{MeV}$ for a few isolated kinematical configurations [18-21]. Comparison of those data and of the data taken for a few configurations at $200 \mathrm{MeV}$ [22] with the theoretical predictions [23,24] shows a mixed picture, similar to the one observed for elastic scattering. Sometimes the agreement between experiment and theory is improved by including $3 \mathrm{NF}$; 
in some cases the $3 \mathrm{NF}$ effects are negligible, and there are also cases in which inclusion of $3 \mathrm{NF}$ drives the prediction off the data.

Measurement of the ${ }^{1} \mathrm{H}(\vec{d}, p p) n$ breakup reaction at the beam energy of $130 \mathrm{MeV}$, performed at KVI Groningen, was designed to provide accurate experimental data in a large fraction of the phase space. Results obtained for cross sections $[25,26]$ allowed one to reliably test predictions of various theoretical approaches. The cross-section data revealed both $3 \mathrm{NF}$ effects and influences of Coulomb interactions. This now has to be further tested in the sector of polarization observables. The ${ }^{1} \mathrm{H}(\vec{d}, p p) n$ process with transversally polarized deuterons gives access to two vector and three tensor analyzing powers, some of which vanish in the case of the elastic scattering. Simple analysis of breakup data [27] led to extracting onetensor analyzing power in a spherical representation, $\operatorname{Re} T_{20}$, for which predicted effects of $3 \mathrm{NF}$ are generally small. This study shows a good description of the data by the $N N$ interaction alone but reveals also certain regions where discrepancies cannot be cured by the inclusion of any of the existing $3 \mathrm{NF}$ models. It is important to mention here that very recently the problems with describing polarization observables was also demonstrated in the data measured for the $p$ - $d$ breakup at $135 \mathrm{MeV}$ [28] and $190 \mathrm{MeV}$ [29], as well as in the case of the $d-p$ breakup data: at $270 \mathrm{MeV}$ [30] and in several configurations at $135 \mathrm{MeV} / \mathrm{A}$ [31].

This article presents a set of data for vector $A_{x}$ and $A_{y}$ and tensor $A_{x x}, A_{x y}$ and $A_{y y}$ analyzing powers collected in the large phase-space region of the ${ }^{1} \mathrm{H}(\vec{d}, p p) n$ breakup reaction at a beam energy of $130 \mathrm{MeV}$. Section II is devoted to definition of the coordinate systems and studied observables. Experimental details, with the focus on improvements with respect to the first run of the read-out electronics and data-acquisition system, are given in Sec. III. In Sec. IV the data-analysis procedure is outlined and the experimental uncertainties are discussed. Theoretical approaches providing predictions which are compared with the data are recalled in Sec. V. The results and their comparison with the theoretical calculations are presented in Sec. VI, and the conclusions are given in Sec. VII.

\section{ANALYZING POWERS OF THE BREAKUP REACTION}

The yield $I(\xi)$ of a reaction leading to a three-particle final state, in the case of an unpolarized target and polarized beam of spin 1 particles, can be expressed in the Cartesian coordinate system by the reaction yield for an unpolarized beam, $I_{o}(\xi)$, vector analyzing powers $A_{x}(\xi), A_{y}(\xi)$, and $A_{z}(\xi)$ and tensor analyzing powers $A_{x y}(\xi), A_{y z}(\xi), A_{x z}(\xi), A_{x x}(\xi)$, $A_{y y}(\xi)$, and $A_{z z}(\xi)$. $\xi$ represents a set of kinematical variables, which for the deuteron-proton breakup can be chosen as $\xi=\left(\theta_{1}, \theta_{2}, \varphi_{12}=\varphi_{1}-\varphi_{2}, S\right)$, i.e., the proton emission angles (see Fig. 1) and the variable $S$, denoting the arc length along the kinematical curve, with 0 set at the minimal energy $E_{2}$ of the second proton. In our studies of the ${ }^{1} \mathrm{H}(\vec{d}, p p) n$ reaction, the deuteron beam is transversally polarized. In this case, shown in Fig. 1, some terms of the general formula given in Ref. [32] vanish, leading to a simpler dependence of $I(\xi)$

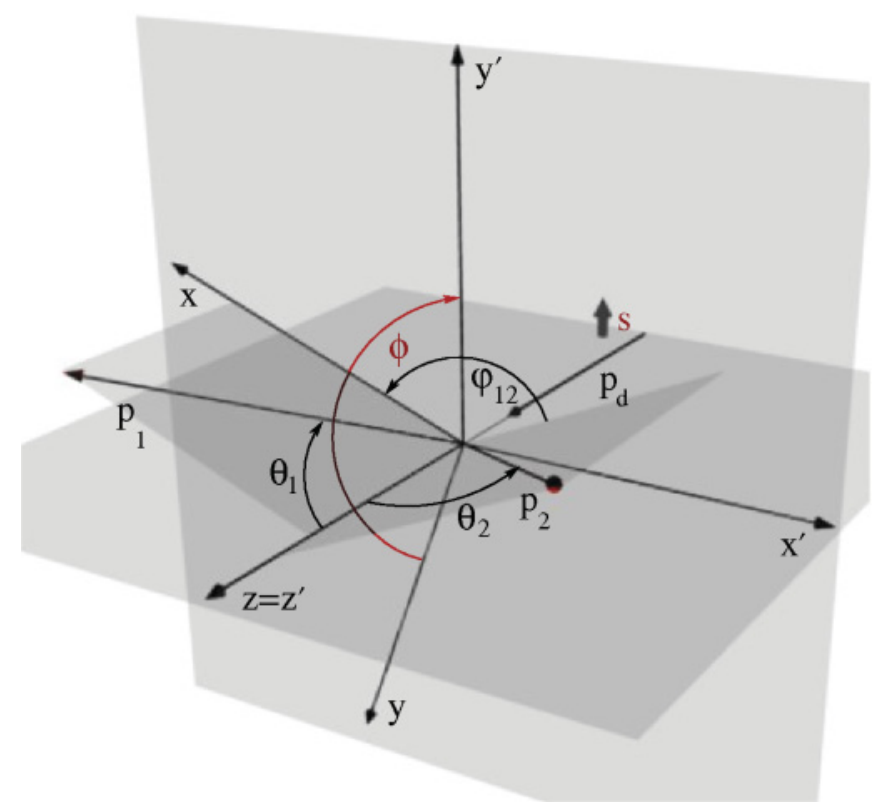

FIG. 1. (Color online) Definition of the coordinate systems for the studied breakup reaction with transversally polarized deuterons: The laboratory system $\left(x^{\prime}, y^{\prime}, z^{\prime}\right)$, with $z^{\prime}$ along the beam momentum direction $\left(\mathrm{p}_{d}\right)$ and $y^{\prime}$ vertically upward, is used to define the angular configuration of the outgoing protons (with momenta $p_{1}$ and $p_{2}$ ). The reaction coordinate system is defined according to Ref. [32], with $z$ along the beam momentum direction and $x$ obtained by projection of $\mathrm{p}_{1}$ onto the plane perpendicular to $z$. The angle $\phi$ is defined as the angle between the $y$ axis and the spin quantization axis $s$, which in the studied case is vertical (parallel to $y^{\prime}$ ).

on the two vector and three tensor analyzing powers:

$$
\begin{aligned}
I(\xi, \phi)= & I_{o}(\xi) \cdot\left\{1+\frac{3}{2} P_{Z}\left[-\sin \phi A_{x}(\xi)+\cos \phi A_{y}(\xi)\right]\right. \\
& +\frac{1}{2} P_{Z Z}\left[\sin ^{2} \phi A_{x x}(\xi)+\cos ^{2} \phi A_{y y}(\xi)\right. \\
& \left.\left.-\sin 2 \phi A_{x y}(\xi)\right]\right\}
\end{aligned}
$$

where $P_{Z}$ and $P_{Z Z}$ denote, respectively, vector and tensor polarization of the beam, both defined with respect to a quantization's axis $s$, and the angle $\phi$ is an angle between the axis $s$ and $y$ axis of the reaction coordinate system (for definition cf. Fig. 1).

The parity conservation of the strong interaction requires that the analyzing powers from Eq. (1) fulfill the relation:

$$
A_{i}\left(\tilde{\xi}, \varphi_{12}\right)=(-1)^{l} A_{i}\left(\tilde{\xi},-\varphi_{12}\right)
$$

where $\tilde{\xi}$ denotes all kinematical variables but the relative azimuthal angle between the proton momenta $\varphi_{12}$, while $l=0$ for $A_{y}, A_{x x}$, and $A_{y y}$ and $l=1$ for $A_{x}$ and $A_{x y}$. The observables that are odd with respect to $\varphi_{12}, A_{x}$ and $A_{x y}$, have to vanish for coplanar configurations. This is also true for the elastic-scattering process in particular. 
Formula (1) can be written in an equivalent way in spherical coordinates [32]:

$$
\begin{aligned}
I(\xi, \phi)= & I_{0}(\xi) \cdot\left\{1+\sqrt{3} P_{Z}\left[\sin \phi \operatorname{Im} i T_{11}(\xi)\right.\right. \\
& \left.+\cos \phi \operatorname{Re} i T_{11}(\xi)\right]-\frac{1}{4} P_{Z Z}\left[\sqrt{2} \operatorname{Re} T_{20}(\xi)\right. \\
& \left.\left.+2 \sqrt{3} \cos 2 \phi \operatorname{Re} T_{22}(\xi)+2 \sqrt{3} \sin 2 \phi \operatorname{Im} T_{22}(\xi)\right]\right\}
\end{aligned}
$$

The terms coming with $\sin \phi$ and $\sin 2 \phi$ vanish for coplanar configurations of the outgoing particles, therefore, especially, for elastic scattering. In this work, we will use the Cartesian representation for breakup observables but the spherical one for elastic-scattering observables.

\section{EXPERIMENTAL SETUP}

The experiment was carried out at Kernfysish Versneller Instituut (KVI) in Groningen, The Netherlands, with the use of the vector- and tensor-polarized deuteron beam accelerated to the energy of $130 \mathrm{MeV}$.

\section{A. Production of polarized deuteron beam}

A beam of deuterons, vector and tensor polarized in the transversal direction, was produced in an atomic beam-type polarized ion source POLIS [33]. The following magnetic fields can be used individually or in various combinations to induce various polarization states of the deuteron beam: SF1 (strong field 1), SF2 (strong field 2), MF (medium field), and WF (weak field). In the experiment described here seven combinations of different magnetic fields (causing different hyperfine transitions) were used, delivering various polarization states described by vector $P_{Z}$ and tensor $P_{Z Z}$ polarizations. The combinations of fields and the maximal values of polarization, corresponding to $100 \%$ efficient transitions between the states of atomic deuterium, are listed in Table I. The polarization states were changed sequentially in a programed cycle. Each state was set for $5 \mathrm{~min}$, while switching between subsequent states took a few seconds. Information about the polarization state was coded as a bit pattern and sent into the data stream.

A beam of polarized deuterons was injected from POLIS to the superconducting cyclotron AGOR and accelerated to the required energy of $130 \mathrm{MeV}$. The beam was guided through the beam line to the experimental setup and focused on the liquid hydrogen target [34]. Further it was transported to the Faraday cup, where the beam current was measured. In the ${ }^{1} \mathrm{H}(\vec{d}, p p) n$ breakup measurement very low current of about $50 \mathrm{pA}$ was used.

\section{B. SALAD detection system}

The polarized deuteron beam was impinging onto the liquid hydrogen target $\left(\mathrm{LH}_{2}\right)$ placed in vacuum, approximately in the center of the scattering chamber. The charged reaction products: two outgoing protons from the breakup process or proton and deuteron from the elastic scattering, were detected in the Small Angle Large Acceptance Detector [35] (SALAD), shown schematically in Fig. 2. The SALAD detection system allowed us to register particles for the polar angles $\theta$ between
TABLE I. Set of the polarization states used in the ${ }^{1} \mathrm{H}(\vec{d}, p p) n$ breakup experiment. The maximum polarizations $P_{Z}, P_{Z Z}$ (for $100 \%$ efficiency of transitions in the ion source) and corresponding combinations of the magnetic fields are shown. The $\mathrm{x}$ indicates that the magnetic field is switched on, whereas the-indicates that the magnetic field is switched off. $I_{f}$ denotes the full beam intensity. In

\begin{tabular}{|c|c|c|c|c|c|c|}
\hline \multirow{2}{*}{\multicolumn{2}{|c|}{$\begin{array}{c}\text { Polarization } \\
\text { states }\end{array}$}} & \multicolumn{4}{|c|}{ Magnetic fields } & \multirow{3}{*}{$\begin{array}{l}\text { Beam } \\
\text { intensity }\end{array}$} \\
\hline & & \multirow{2}{*}{ SF1 } & \multirow{2}{*}{ SF2 } & \multirow{2}{*}{$\mathrm{MF}$} & \multirow{2}{*}{ WF } & \\
\hline$P_{Z}$ & $P_{Z Z}$ & & & & & \\
\hline 0 & 0 & - & - & - & - & $I_{f}$ \\
\hline$+\frac{1}{3}$ & +1 & $\mathrm{x}$ & - & - & - & $I_{f}$ \\
\hline$+\frac{1}{3}$ & -1 & - & $\mathrm{x}$ & - & - & $I_{f}$ \\
\hline 0 & +1 & $\mathrm{X}$ & - & $\mathrm{X}$ & - & $\frac{2}{3} I_{f}$ \\
\hline 0 & -2 & - & $\mathrm{X}$ & $\mathrm{X}$ & - & $\frac{2}{3} I_{f}$ \\
\hline$+\frac{2}{3}$ & 0 & $\mathrm{x}$ & $\mathrm{X}$ & - & - & $I_{f}$ \\
\hline$-\frac{2}{3}$ & 0 & - & - & - & $\mathrm{X}$ & $I_{f}$ \\
\hline
\end{tabular}
the case of transitions with medium field on, the beam intensity is reduced to $2 / 3$ of $I_{f}$ in the case of $100 \%$ efficient transitions.

$10^{\circ}$ and $35^{\circ}$ and for the full range of the azimuthal angles $\varphi$, from $0^{\circ}$ to $360^{\circ}$. The implementation of this detector to the breakup experiment is described in Ref. [25]; here we present only briefly its main features.

The charged particles emitted from the target, after leaving the vacuum chamber through a thin exit window, were passing the MultiWire Proportional Chamber (MWPC), transmission $\Delta E$ detectors and were stopped in the $E$ detectors. The MWPC detector was used for reconstruction of the charged particle emission angles angles with the overall accuracy of $0.3^{\circ}$ for the polar angle and between $0.6^{\circ}$ and $3.0^{\circ}$ for the azimuthal angle. The $E$ slabs were arranged vertically and $\Delta E$ strips were oriented horizontally; in this way a two-dimensional array of $140 \Delta E-E$ telescopes was created. It allowed to

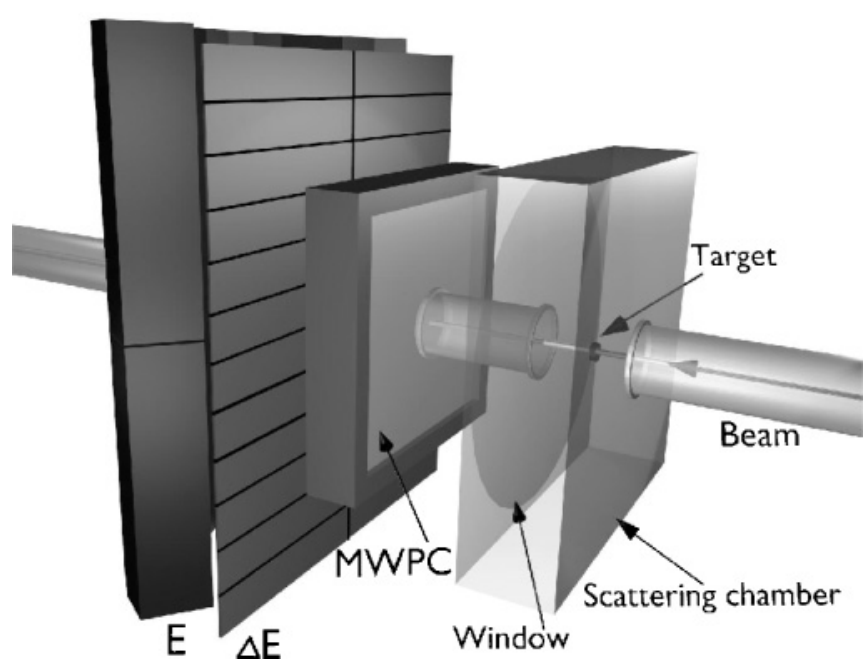

FIG. 2. Schematic view of the experimental setup. The beam direction, the position of the target in the scattering chamber, the MultiWire Proportional Chamber (MWPC) and the $\Delta E$ and $E$ detectors are shown. 


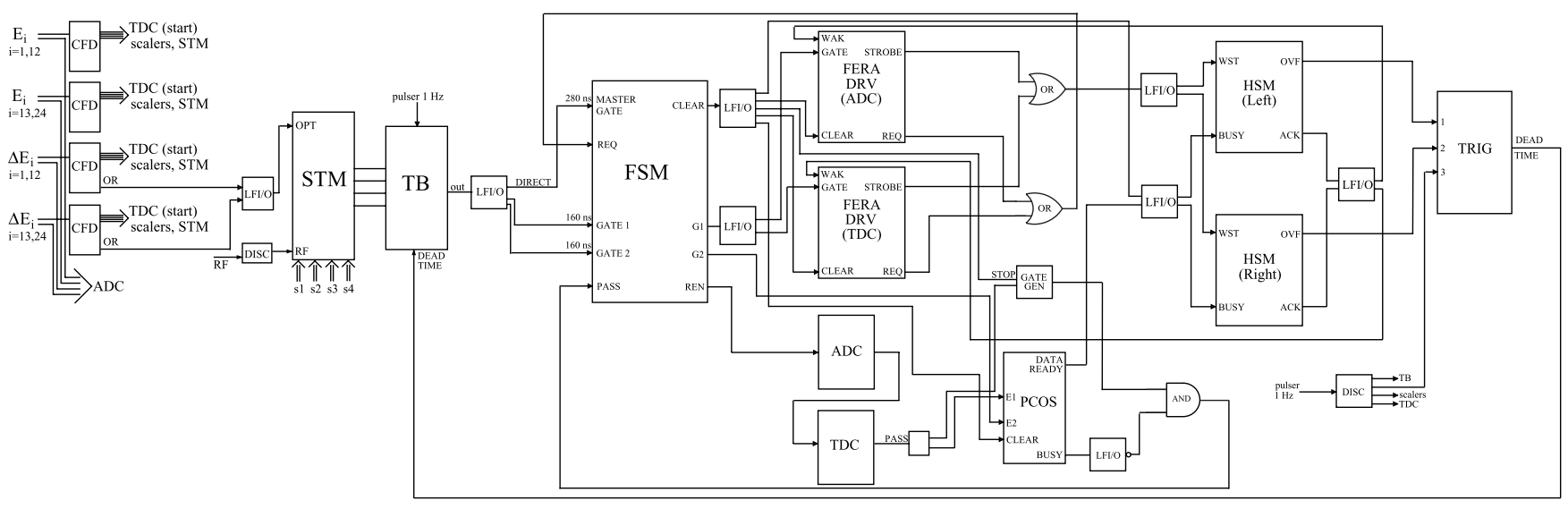

FIG. 3. A schematic diagram of the electronics circuit.

identify particles, to define trigger conditions, and to determine energies of the particles. The energy resolution of the $E$ counters was about $5 \%$.

As compared to the first run, of which results were published in Refs. [25,26], a few changes were introduced in the experimental setup. Among them, the distance between the $\Delta E-E$ wall and the target was increased by about $15 \%$ in order to use the $\Delta E-E$ wall in full, while in the first run it was slightly in the shadow of the MWPC frame. The most important changes were done, however, in the domain of the read-out and data-acquisition system, as described in the following subsection.

\section{Electronics, read-out, and data acquisition}

The layout of the electronic circuit is presented in Fig. 3. All parts of the trigger electronics and signal processing up to read-out were very similar to the ones used in the first experimental run. Triggers were constructed in the SALAD Trigger Module (STM) [36] and downscaled in the Trigger Box (TB) module. In this way contribution of coincidences was enhanced (in the sense of the trigger rate) with respect to single events. Detailed description of this part of the electronics can be found in Ref. [25].

A new, important feature of the system, which lead to a substantially improved data throughput, about 6 times as compared to the first run, was decoupling of the read-out process, when the data were written to one of the two High Speed Memories (HSM), and the processor-controlled transfer of the data from memory to tape. The control over read-out was performed by the FERA System Manager (FSM), which (if not busy with a previous trigger) distributed the trigger signal as a gate to the Proportional Chamber Operating System (PCOSIII), as gates for signal integration in a set of ADC's and as common stop signals for a set of TDC's. The details on the FERA system with distributed gates, realized with the use of the Tagger and Extender modules, can be found in Ref. [37].

In the read-out process, the control over the FERA bus was consecutively overtaken by all the modules in the "readoutenable/pass" chain, which subsequently sent their conversions to one of the HSM modules. The handshaking was done by the strobe/acknowledge mechanism between the module currently controlling the FERA bus and the HSM (via the FERA Driver), without any control from the side of the front-end processor. With the FERA (ADC and TDC) transmission completed, the PCOS data were sent to the same memory via the PCOS ECL bus. Switching between the two data busses, the FERA Data Bus and the bus of PCOS, was done in the Data Selector Module, steered with the PCOS Busy signal. After all the data were sent to the memory, the FERA digitizing modules and the PCOS latching modules were cleared and the system was ready for acquiring a new event. Up to that moment it was blocked against new triggers and the corresponding dead time (the ratio of numbers of lost and all events) was monitored in scalers.

The front-end processor, RIO2 with LynxOS real time operating system, controlled the data acquisition with the use of the Multi-Branch System (MBS) [38]. The processor was hosted in a VME Crate, together with the HSM modules and the so-called Trigger module. When the currently active HSM was almost filled, it generated an overflow signal which caused a processor interrupt. As a reaction to that interrupt the processor took care to disable (after completed full event read out) the active HSM module and enable the other one. For the time of switching the memories, the dead-time signal was sent to the TB module to block it against producing the triggers. In order to determine the corresponding dead time, the output of pulser producing signals with a frequency of $1 \mathrm{kHz}$ was also sent to scalers via TB. When the new memory had become enabled, the triggers were released and, with the data accumulation active, the processor read the filled memory content, directing the data to the DLT tape and sending its fraction to the on-line analysis for monitoring purposes.

\section{DATA ANALYSIS}

\section{A. Event selection and energy calibration}

The events of interest were coincidences of two charged particles, i.e., proton-proton pairs from the breakup process or, necessary for determination of the beam polarization, deuteron-proton pairs from the elastic scattering. Events with only one charged particle, proton or deuteron, registered in SALAD (single events) were also recorded with a strongly suppressed rate. Particle identification was based on the $\Delta E-E$ technique, providing for all telescopes and in the whole energy 
range an excellent separation between protons and deuterons. In addition to the particle identification cuts set on the $\Delta E-E$ spectra, the limits were also set on the time spectra of all $\Delta E$ and $E$ detectors, with the aim to reduce contribution of accidental coincidences: only events registered in a 20-ns-wide time window were accepted.

In order to obtain the number of events from the breakup reaction as a function of proton energy or, equivalently, as a function of the arc length $S$ along the kinematical curve, energy calibration is required. For that purpose elastic-scattering events, measured during special calibration runs with energy degraders positioned in front of the scintillation hodoscope, were used. The positions of the peaks corresponding to protons from the elastic scattering which traversed the degraders were compared with the results of simulations taking into account all energy losses of protons. In the first step a linear function was fitted to the relation of the deposited energy versus pulse height. Afterward also a small correction for nonlinearity in this relation was introduced. Such nonlinearity is caused by light quenching in the scintillating material and can be described by Birks formula [39] or by its version enclosing higher-order effects, introduced by Chou [40]. However, it has been found that the effects of nonlinearity are small: the linear channel-energy relation had to be modified only below $30 \mathrm{MeV}$. Since the departure from linearity reached at most $1.5 \mathrm{MeV}$, the correction was introduced by only a slight change of the slope of the linear dependence below $30 \mathrm{MeV}$. In the last step of calibration, the relation between the energy deposited by protons in the $E$ counter and their energy at the moment of reaction was found by Monte Carlo simulation of the energy losses. More details concerning procedures of event selection and energy calibration can be found in Refs. [25] and [41].

\section{B. Determination of the beam polarization}

Values of vector and tensor polarization of the deuteron beam were determined from analysis of the elastic-scattering events. For $\theta_{d}^{\text {c.m. }} \leqslant 119^{\circ}$ the values of the analyzing powers of the elastic scattering were determined in a dedicated experiment at RIKEN [16], using absolute calibration of the beam polarization via ${ }^{12} \mathrm{C}(\vec{d}, \alpha){ }^{10} \mathrm{~B}^{*}\left[2^{+}\right]$reaction [42]. The angular region covered by that experiment overlaps with the acceptance of the SALAD detector for $d$ - $p$ coincidences at exactly one point. The procedure of the polarization determination based on analysis of elastic scattering at only one angle is described in Ref. [17]. However, thanks to very good particle identification, also single deuterons registered in SALAD can be used for determining the rates for elastic scattering. This opens a way for using the whole experimentally measured distributions from both experiments and for reducing the uncertainty of the resulting beam polarizations.

With the purpose to determine beam polarization, elasticscattering events collected for the selected polarization state $P \equiv\left(P_{Z}, P_{Z Z}\right)$ were sorted into bins of $1^{\circ}$ in the laboratory polar angle: proton angle $\theta_{p}$ in the case of $d$-p coincidences, or deuteron angle $\theta_{d}$ in the case of single deuterons. They were sorted also with respect to the angle $\phi$ (cf. Fig. 1) with the binning of $10^{\circ}$. The obtained numbers of events have been normalized to the beam current collected in the Faraday cup and corrected for the dead-time losses. After normalization and transformation of polar angles to the center-of-mass system, the rates of the elastically scattered events $N_{P}^{\theta_{d}^{\text {c.m. }}}(\phi)$ for the selected polarization state $P$ were obtained. On the basis of these numbers the ratio:

$$
f_{P}^{\theta_{d}^{\text {c.m. }}}(\phi)=\frac{N_{P}^{\theta_{d}^{\text {c.m. }}}(\phi)-N_{0}^{\theta_{d}^{\text {c.m. }}}(\phi)}{N_{0}^{\theta_{d}^{\text {c.m. }}}(\phi)}
$$

was constructed. $N_{0}^{\theta_{d}^{\text {c.m. }}}(\phi)$ denotes the analogously obtained rate of events for an unpolarized beam. All factors constant in time, as, e.g., target thickness or detection efficiency, cancel in the ratio, and thus do not have to be included in the normalization procedure.

The evaluated numbers of events are directly proportional to the corresponding reaction yields. Using Eq. (3) limited to terms nonvanishing in the case of elastic scattering, the formula for $N_{P}^{\theta d^{c . m .}}$ can be expressed by:

$$
\begin{aligned}
N_{P}^{\theta_{d}^{\text {c.m. }}}(\phi)= & N_{0}^{\theta_{d}^{\text {c.m. }}} \cdot \kappa\left[1+i T_{11}^{\mathrm{el}}\left(\theta_{d}^{\mathrm{c} . \mathrm{m} .}\right) \sqrt{3} P_{Z} \cos \phi\right. \\
& -T_{22}^{\mathrm{el}}\left(\theta_{d}^{\text {c.m. }}\right) \frac{\sqrt{3}}{2} P_{Z Z} \cos 2 \phi \\
& \left.-T_{20}^{\mathrm{el}}\left(\theta_{d}^{\text {c.m. }}\right) \frac{\sqrt{2}}{4} P_{Z Z}\right]
\end{aligned}
$$

where $\kappa$ denotes the ratio of normalization factors for the two states, which should be equal to 1 in the case of perfect normalization, and $i T_{11}^{\mathrm{el}}, T_{22}^{\mathrm{el}}, T_{20}^{\mathrm{el}}$ are spherical vector- and tensor analyzing powers of elastic scattering. Substituting formula (5) into equation (4) results in the final expression for the ratio of rates:

$$
\begin{aligned}
f_{P}^{\theta_{d}^{\text {c.m. }}}(\phi)= & \kappa\left[1+i T_{11}^{\mathrm{el}}\left(\theta_{d}^{\text {c.m. }}\right) \sqrt{3} P_{Z} \cos \phi\right. \\
& -T_{22}^{\mathrm{el}}\left(\theta_{d}^{\text {c.m. }}\right) \frac{\sqrt{3}}{2} P_{Z Z} \cos 2 \phi \\
& \left.-T_{20}^{\mathrm{el}}\left(\theta_{d}^{\text {c.m. }}\right) \frac{\sqrt{2}}{4} P_{Z Z}\right]-1 .
\end{aligned}
$$

This is a function of the type:

$$
f_{P}^{\theta_{d}^{\text {c.m. }}}(\phi)=a \cdot \cos (\phi)+b \cdot \cos (2 \phi)+c,
$$

which can therefore be fitted to the ratio of the experimental rates. Using the fit results for angle $\theta_{d}^{\text {c.m. }}=119^{\circ}$ and experimental values of the analyzing powers for elastic scattering, $i T_{11}^{\mathrm{el}}, T_{22}^{\mathrm{el}}$, and $T_{20}^{\mathrm{el}}$, at this angle, the values of $P_{Z}, P_{Z Z}$, and $\kappa$ can be determined. The refined version of this procedure takes into account the whole measured angular distributions and, therefore, leads to a reduction of the statistical uncertainties, while simultaneously also the consistency of both measurements is checked. Details concerning the way of comparing distributions measured at RIKEN and in this experiment can be found in Ref. [41]. The results obtained in this way for various polarization states and their statistical uncertainties are given in Table II. Systematic errors of the polarization values are mainly due to systematic uncertainties of the reference analyzing powers [16] and are estimated to be around 3\%. 
TABLE II. Beam polarization values and their statistical uncertainties obtained for various states; for more details see text.

\begin{tabular}{lrcrccc}
\hline \hline$P$ & \multicolumn{1}{c}{$P_{Z}$} & $\Delta P_{Z}$ & \multicolumn{1}{c}{$P_{Z Z}$} & $\Delta P_{Z Z}$ & $\kappa$ & $\Delta \kappa$ \\
\hline$\left(+\frac{1}{3},-1\right)$ & 0.256 & 0.002 & -0.757 & 0.005 & 1.00 & 0.01 \\
$\left(+\frac{2}{3}, 0\right)$ & 0.449 & 0.003 & -0.118 & 0.005 & 1.00 & 0.01 \\
$\left(-\frac{2}{3}, 0\right)$ & -0.444 & 0.003 & 0.050 & 0.005 & 0.99 & 0.01 \\
$(0,+1)$ & -0.068 & 0.003 & 0.556 & 0.005 & 1.06 & 0.01 \\
$(0,-2)$ & 0.021 & 0.002 & -1.340 & 0.005 & 1.06 & 0.01 \\
$\left(+\frac{1}{3},+1\right)$ & 0.198 & 0.003 & 0.672 & 0.006 & 0.99 & 0.01 \\
\hline \hline
\end{tabular}

It is necessary to point out that, with a correct relative normalization between beam polarization states, one should obtain $\kappa$ value which is statistically consistent with 1 . As is shown in Table II, this was not always true. The reason for small departures from 1 can be attributed to the systematic error in measurements of a very low beam current. This problem is discussed in more detail in subsection IV D1.

\section{Evaluation of the vector and tensor analyzing powers for the breakup reaction}

The data were analyzed for a set of kinematical configurations of the two outgoing protons spanned on a grid of polar angles $\theta_{1}, \theta_{2}$ between $15^{\circ}$ and $30^{\circ}$ in steps of $5^{\circ}$. The absolute values of relative azimuthal angles $\varphi_{12}$ were varied between $0^{\circ}$ (for $\theta_{1} \neq \theta_{2}$ ) or $20^{\circ}$ (for $\theta_{1}=\theta_{2}$ ) and $180^{\circ}$ with the step of $20^{\circ}$. For each configuration with $\varphi_{12}$ from $20^{\circ}$ to $160^{\circ}$ its "mirror configuration" with negative $\varphi_{12}$, has been analyzed.

In the first step, for each combination of angles $\theta_{1}, \theta_{2}, \varphi_{12}$ and for each polarization state the kinematical spectra $E_{2}$ versus $E_{1}$ were built (see example in Fig. 4). The angular ranges of event integration were chosen to be $\Delta \theta_{1}=\Delta \theta_{2}=4^{\circ}$ and $\Delta \varphi_{12}=20^{\circ}$. This corresponds to 8 times larger solid angle as compared to the cross-section analysis [25] and allows to achieve good statistical accuracy of individual data points without smearing out details of the angular distributions. Then, events on the kinematical curve were divided into bins in $S$ with the width of $8 \mathrm{MeV}$ (shaded area in Fig. 4).

The procedure for subtracting the (low) background underlying the data is described in detail in Ref. [25]. The obtained number of events have been normalized to the beam current collected in the Faraday cup and corrected for dead-time losses. After normalization, the rates of events $N_{P}^{\xi}$ for the selected polarization state $P$ and kinematical point $\xi=\left(\theta_{1}, \theta_{2}, \varphi_{12}, S\right)$ were obtained and the ratio

$$
f_{P}^{\xi}(\phi)=\frac{N_{P}^{\xi}(\phi)-N_{0}^{\xi}(\phi)}{N_{0}^{\xi}(\phi)}
$$

was constructed. $N_{0}^{\xi}$ denotes the rate for the unpolarized beam. Applying the Eq. (1), $f_{P}^{\xi}$ can be written as

$$
\begin{aligned}
f_{P}^{\xi}(\phi)= & \kappa\left[1-\frac{3}{2} \sin \phi P_{Z} A_{x}(\xi)+\frac{3}{2} \cos \phi P_{Z} A_{y}(\xi)\right. \\
& +\frac{1}{2} \sin ^{2} \phi P_{Z Z} A_{x x}(\xi)+\frac{1}{2} \cos ^{2} \phi P_{Z Z} A_{y y}(\xi) \\
& \left.-\frac{1}{2} \sin 2 \phi P_{Z Z} A_{x y}(\xi)\right]-1
\end{aligned}
$$

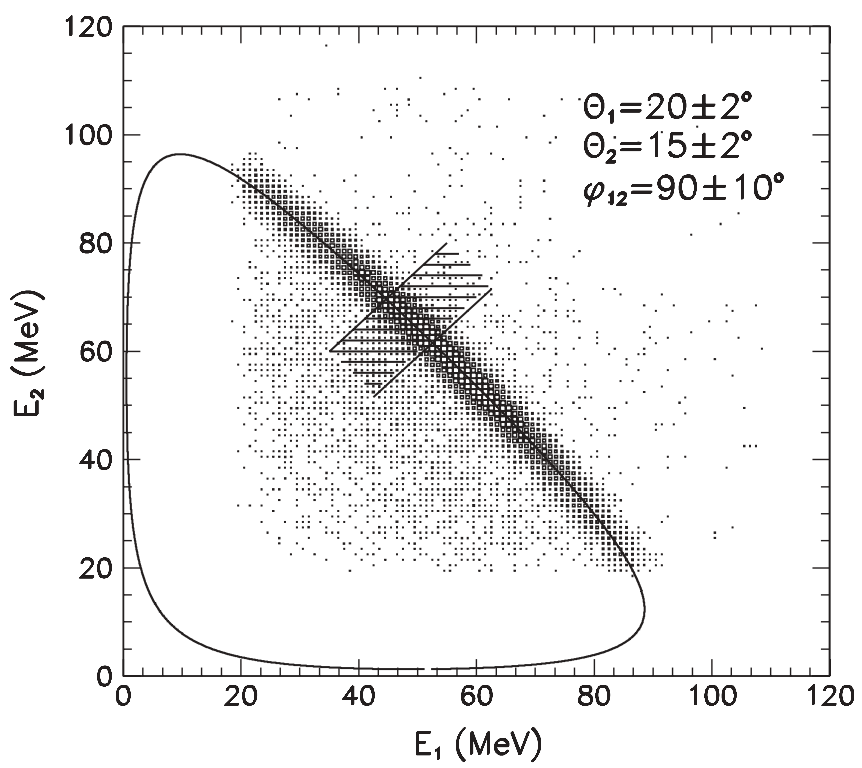

FIG. 4. An example of the $E_{2}$ versus $E_{1}$ energy spectrum for selected angular configuration of the breakup process $\left(\theta_{1}=20^{\circ}, \theta_{2}=\right.$ $15^{\circ}, \varphi_{12}=90^{\circ}$ ). A sample $\Delta S$ bin is marked as a shaded area.

The polarizations $P_{Z}$ and $P_{Z Z}$, as well as $\kappa$ values are known from the analysis of elastic scattering (cf. Table II). Vector and tensor analyzing powers were extracted from the fit of the function (9) to the experimental distributions of $f_{P}^{\xi}(\phi)$. Examples of such distributions with the fitted curves, obtained for two mirror configurations, are shown in the two upper panels of Fig. 5. In certain configurations characterized by low cross sections, the statistical uncertainties of $f_{P}^{\xi}(\phi)$ were large. The points with uncertainty exceeding 0.5 were rejected from the analysis, since they do not set any significant constraints on asymmetry values. On the other hand, in geometries with low $\varphi_{12}$ values, acceptance losses can occur due to registering both protons in the same detector segment. For these two reasons, the number of points for the fit of $f_{P}^{\xi}(\phi)$ was not always equal. For the purpose of keeping the number of degrees of freedom at a reasonable level, the fit was performed provided at least 12 data points were available.

In order to decrease statistical errors of the results, the analyzing power values obtained from the fits of Eq. (9) for the kinematical configurations with $\pm \varphi_{12}$ were combined together according to the parity rules [see Eq. (2)]:

$$
A_{i}(\xi)=\frac{1}{2}\left[A_{i}\left(\tilde{\xi}, \varphi_{12}\right)+(-1)^{k} A_{i}\left(\tilde{\xi},-\varphi_{12}\right)\right],
$$

where $k=0,1$ for the even and odd observables, respectively. For the purpose of testing the data consistency, the observables can be combined also in the following way:

$$
O_{i}(\xi)=A_{i}\left(\tilde{\xi}, \varphi_{12}\right)-(-1)^{k} A_{i}\left(\tilde{\xi},-\varphi_{12}\right) .
$$

Such combinations, in turn, should be consistent with 0 within their statistical errors. Obviously, both ways of combining analyzing powers do not apply to the coplanar configurations of the outgoing protons, which transform onto themselves when reversing the sign of $\varphi_{12}$.

In the approach described above, which will be further called "free fit," the constraints set by parity conservation 


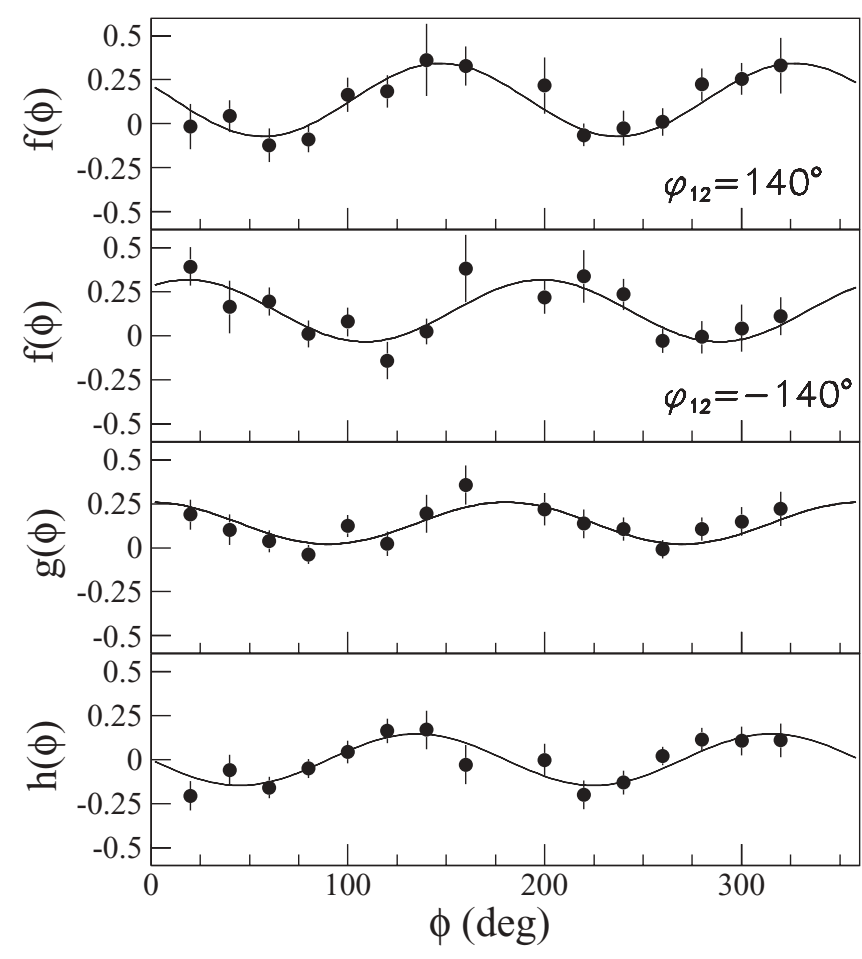

FIG. 5. Examples of asymmetry distributions obtained for pure tensor polarization state $P=(0,-2)$, for two (mirror) kinematical configurations with $\theta_{1}=25^{\circ}, \theta_{2}=20^{\circ}, \varphi_{12}= \pm 140^{\circ}$, and $S=$ $100 \mathrm{MeV}$. In the two top panels asymmetries $f_{P}^{\xi}(\phi)$ for these configurations are presented, and in the two bottom panels their linear combinations, $g_{P}^{\xi}(\phi)$ and $h_{P}^{\xi}(\phi)$ (see text), are shown. The error bars represent statistical uncertainties. The lines result from the fit of the corresponding functions, as described in the text.

are used only in the very last step of the analysis, when the analyzing powers obtained for mirror configurations are combined according to Eq. (10). Alternatively, the parity conservation rules had been applied in an earlier stage, before the fit was performed. The following combinations of asymmetries for mirror configurations $\left(\tilde{\xi}, \varphi_{12}\right)$ and $\left(\tilde{\xi},-\varphi_{12}\right)$ were obtained:

$$
g_{P}^{\xi}(\phi)=\frac{f_{P}^{\tilde{\xi}, \varphi_{12}}(\phi)+f_{P}^{\tilde{\xi},-\varphi_{12}}(\phi)}{2}
$$

and

$$
h_{P}^{\xi}(\phi)=\frac{f_{P}^{\tilde{\xi}, \varphi_{12}}(\phi)-f_{P}^{\tilde{\xi},-\varphi_{12}}(\phi)}{2} .
$$

Using Eqs. (9) and (2), such linear combinations can be expressed as

$$
\begin{aligned}
g_{P}^{\xi}(\phi)= & \kappa\left[1+\frac{3}{2} \cos \phi P_{Z} A_{y}(\xi)+\frac{1}{2} \sin ^{2} \phi P_{Z Z} A_{x x}(\xi)\right. \\
& \left.+\frac{1}{2} \cos ^{2} \phi P_{Z Z} A_{y y}(\xi)\right]-1
\end{aligned}
$$

and

$$
h_{P}^{\xi}(\phi)=\kappa\left[-\frac{3}{2} \sin \phi P_{Z} A_{x}(\xi)-\frac{1}{2} \sin 2 \phi P_{Z Z} A_{x y}(\xi)\right] .
$$

Fit of the function (14) to the experimentally determined distribution $g_{P}^{\xi}(\phi)$ leads to determination of the even analyzing powers, while the odd ones are obtained from the fit of the function (15) to $h_{P}^{\xi}(\phi)$. Examples of fits of these functions to the data are shown in the two lower panels of Fig. 5. The procedure based on the fit of such linear combinations of asymmetries will be further called "parity-constrained fit."

Results of both methods, free fit and parity-constrained fit, should lead to results consistent with each other. This has been proven to be true within statistical uncertainties. The parity-constrained method of analysis, which avoids superfluous fit parameters, leads to $\chi^{2}$ distributions which can be interpreted in terms of statistical properties. Therefore the results presented in this article are obtained by that method, since this provides more trustful uncertainties of the fitted parameters. The other method was applied only to check the data consistency.

After the fits were performed, consistency of the results obtained for the individual states was checked, as discussed in the following section, and the weighted averages of the analyzing powers extracted for various polarization states were calculated.

\section{Experimental uncertainties}

The experimental uncertainties vary strongly in the presented set of analyzing power data, but still the statistical uncertainties dominate. In this section statistical errors together with possible sources of systematic uncertainties and their estimated magnitudes are discussed.

\section{Statistical errors}

The statistical uncertainties of the analyzing powers comprise the errors from the fit procedure, which are the main contributions, and the statistical errors of the beam polarizations $P_{Z}$ and $P_{Z Z}$ and of the normalization factor $\kappa$ (Table II), following from the fit to elastic-scattering distributions. The resulting statistical uncertainties vary between 0.01 and 0.1 .

\section{Systematic effects and data consistency checks}

The data analysis presented in this article relies on determining ratios of rates measured with polarized and unpolarized beams. It is therefore independent of many experimental factors, like, e.g., detection efficiency of the particles in the MWPC and in the scintillator hodoscope, losses due to particle identification cuts in the $\Delta E-E$ spectra, uncertainties in determination of the solid angles, and so on. The other potential systematic effects, which do not cancel in the ratio, are discussed below. In most cases, analysis of events originating from the elastic-scattering process is an important tool for reducing and controlling the magnitude of these uncertainties.

One of the main contributions to the systematic uncertainty originates from a systematic uncertainty of the polarization values. Following discussion from subsection IV B, we can attribute a relative error of about $3 \%$ to that effect. This influences only the common scaling factors of the distributions.

In spite of a very careful relative normalization of the rates for the polarized and unpolarized beam, the analysis of the elastic scattering reveals some remnant factor $\kappa$. It has been checked that its values are stable in time and do not depend 
on the downscaling factors applied to the triggers. Statistically significant departure of $\kappa$ from 1 , which reflects a systematic error of the normalization procedure, is observed in two pure tensor polarization states. This effect can be attributed to a small current offset, which is difficult to avoid in measuring very low beam currents. For these two states the medium field was switched on, which reduces the average beam current (cf. Table I) and in this way changes the relative contribution of the instrumental offset as compared to the case of the unpolarized state, characterized by the full beam intensity. As a consequence, the ratio (8) is affected. However, since $\kappa$ is a purely luminosity factor, it is independent from the studied process and its values, determined in the fits of distributions of the elastic scattering, are directly applicable for the breakup reaction. Therefore the normalization to luminosity is not expected to cause any systematic uncertainty on the breakup analyzing power results.

The uncertainty in reconstructing the polar and azimuthal angles originates from the resolution given by the distances between the wires of the MWPC and from possible imperfections in the geometry of the setup. The latter source for systematic effects can be controlled very well on the basis of elastic-scattering kinematics [25]. It has been shown that there was no, at the level below $0.3^{\circ}$, shift of the polar angles. Taking into account the ranges of angles accepted for the analysis of the kinematical configurations and the angular resolution, the systematic effects related to the determination of the particle emission angles are negligible. The accuracy of the determination of the azimuthal angle $\varphi$ is, however, not only important for the proper selection of events belonging to a particular kinematical configuration. It is directly related to the accuracy of the angle $\phi$ (cf. Fig. 1) and, in turn, the uncertainty in determining zero of $\phi$ would produce an additional phase $\delta$ in Eq. (9) and affect the fitted analyzing powers. The possible magnitude of $\delta$ can be studied via the elastic-scattering process, for which the terms with odd observables vanish, so the additional phase in functions $\cos (\phi+\delta)$ and $\cos (2 \phi+2 \delta)$ produces forbidden dependency of the $f_{P}^{\theta}(\phi)$ function [cf. Eq. (6)] on $\sin (\phi)$ and $\sin (2 \phi)$. The same holds for coplanar configurations of the breakup process, but in the case of the elastic scattering the statistical errors of the data points are much smaller and the influence of the possible nonzero $\delta$ phase can be studied with much higher sensitivity. We transform Eq. (6) to

$$
\begin{aligned}
f_{P}^{\theta}(\phi)= & \kappa\left\{1+i T_{11}^{\mathrm{el}}(\theta) \sqrt{3} P_{Z} \cos (\phi+\delta)\right. \\
& -T_{22}^{\mathrm{el}}(\theta) \frac{\sqrt{3}}{2} P_{Z Z} \cos [2(\phi+\delta)] \\
& \left.-T_{20}^{\mathrm{el}}(\theta) \frac{\sqrt{2}}{4} P_{Z Z}\right\}-1
\end{aligned}
$$

and treat $\delta$ as an additional free parameter of the fit. The values of $\delta$ resulting from the fit to the data are consistent with 0 for all the polarization states, with the weighted average $\delta=$ $0.36^{\circ} \pm 0.29^{\circ}$. The admixture of "wrong" analyzing powers is suppressed by a factor of the order of $\delta$ in radians, so even for

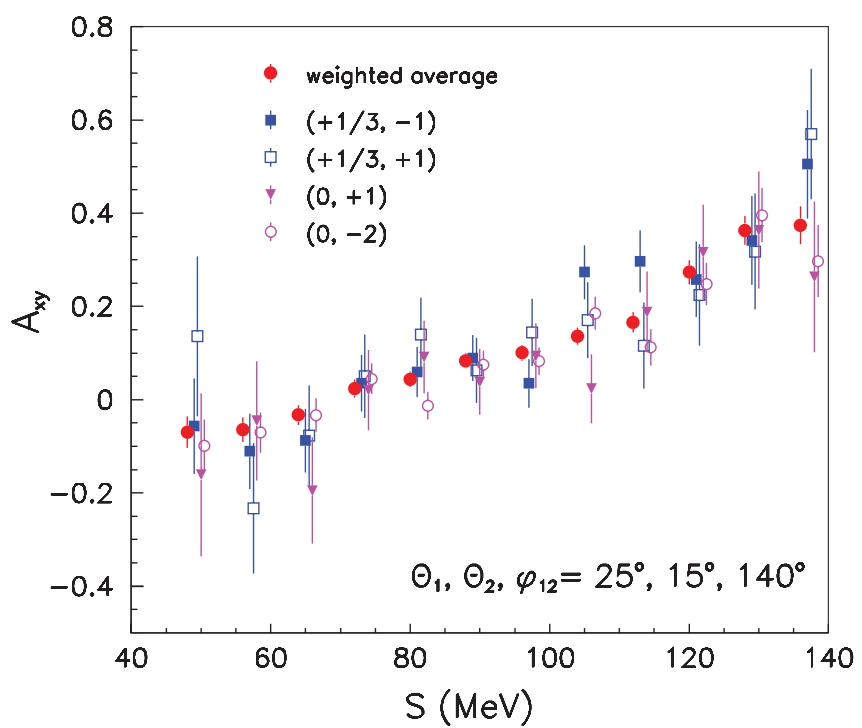

FIG. 6. (Color online) An example of the distribution of $A_{x y}$ versus $S$ for one geometry of the outgoing protons, specified in the panel. The results obtained for all beam states with nonzero tensor polarization (characterized with values of $P_{Z}$ and $P_{Z Z}$ in the legend) are shown together with their weighted average. For clarity, the data points corresponding to individual states have been slightly shifted in $S$.

the largest analyzing power values, their distorting contribution cannot exceed 0.01 .

The events accepted within the gates on the time spectra are true+random coincidences. On the basis of the neighboring peaks in the time spectra it was possible to determine contribution of purely random events which was very small, below $0.3 \%$. When the amounts of random events were subtracted from the numbers of the true+random ones, the numbers of the true events were obtained. However, the ratios $f_{P}^{\xi}$ obtained for the true coincidences and for the true+random ones are almost identical, the observed differences between them are by at least one order of magnitude smaller than their statistical errors. Therefore the uncertainty of the procedure of subtracting accidental events can be neglected.

The overall data consistency can be controlled by checking if the parity rules are fulfilled. This subject is discussed in more detail in Sec. VI. Moreover, consistency of results obtained for individual polarization states has been confirmed; see the example in Fig. 6.

\section{THEORETICAL FORMALISM}

The experimentally determined observables are compared to results of calculations performed with three ways modeling of the interactions in the system of three nucleons. Below the three approaches to treat the $3 \mathrm{~N}$ dynamics are briefly outlined.

\section{A. Realistic $N N$ potentials and $3 N F$ models}

Modern $N N$ forces, so-called realistic potentials, like charge-dependent (CD) Bonn [43], AV18 [44], Nijm I, and Nijm II [45], describe a long-range interaction according to the meson-exchange picture, while the implemented short-range 
part is phenomenological. These potentials can be combined with the models of $3 \mathrm{NF}$, which are refined versions of the force proposed by Fujita and Miyazawa [46]. They considered a process where a pion is exchanged between two nucleons, leading to an excitation of a $\Delta$ isobar, which subsequently decays, exchanging a pion with the third nucleon. The $2 \pi$ exchange TM 3NF model [47] contains one cut-off parameter, $\Lambda_{\mathrm{TM}}$, expressed in units of the pion mass $m_{\pi}$. The value of $\Lambda_{\mathrm{TM}}$ is adjusted for each combination of the $N N$ potential and the TM $3 \mathrm{NF}$ to reproduce the value of the ${ }^{3} \mathrm{H}$ binding energy [48]. The current version of this model, TM99, is consistent with chiral symmetry [49]. When the $3 N$ system is studied with the AV18 NN potential, it is also combined with the Urbana IX $3 \mathrm{NF}$ [50]. This force contains the two-pion exchange contribution due to intermediate $\Delta$ excitation and a phenomenological shorter-range part. The general overviews of the Faddeev formulation of the $3 N$ system with the realistic potentials and $3 \mathrm{NF}$ included are presented in Refs. [51,52].

Recently, studies of relativistic effects in the breakup process including such features as relativistic kinematics and boost potential effects has been presented in Ref. [53]. As dynamical input the CD Bonn potential was taken, thus the calculations neglect any additional $3 N$ dynamics, going beyond this pure $N N$ interaction. At energy studied in this article the predicted relativistic effects are moderate in cross sections and practically negligible in analyzing powers, therefore no comparison of the data with such calculations will be shown.

The Coulomb interaction was for the first time included into the calculation of breakup observables in the approach with two-baryon coupled-channels potential, described briefly in subsection V C. Very recently, this method, with screening and renormalization, has also been applied to purely nucleonic $N N$ and $3 N$ force models, AV18+Urbana IX 3NF [54], but those results are not shown in the present article.

\section{B. Chiral perturbation theory (ChPT)}

Chiral perturbation theory (ChPT) is based on the most general Lagrangian for Goldstone bosons (pions) and matter fields (nucleons) consistent with the (broken) chiral symmetry of the QCD [55,56]. In the framework of ChPT two types of interaction emerge: long-range pion exchanges, with crucial constraints of chiral symmetry, and contact interactions, with the associated low-energy constants (LEC). Due to chiral symmetry a power counting can be devised that enables one to estimate the importance of individual graphs to the $N N$ interaction. For the $N N$ system, due to its nonperturbative character, the problem becomes involved since in principle an infinite number of diagrams contributes at a given order. It was Weinberg [57] who recognized that ChPT can be used to define a nuclear potential, which is then summed to all orders using numerical techniques. In this way, only a finite number of diagrams contributes at a given order and the nonperturbativity of nuclear systems is taken into account. The nuclear potential is expanded in this way in terms of $\left(Q / \Lambda_{\chi}\right)^{v}$, where $Q$ refers to a typical momentum of the nucleons or the pion mass and $\Lambda_{\chi}$ represents the chiral symmetry-breaking scale of $\approx 1 \mathrm{GeV}$. The LEC's at leading-order (LO) and next-to-leading order (NLO) are obtained by fitting the ChPT $N N$ potential predictions to the $N N$ data and three LEC's of the next-to-next-to-leading order (N2LO) potential can be determined from the $\pi N$ scattering data [58]. In next-to-next-to-next-to-leading order (N3LO), there are new short-distance LEC's that need to be fitted to $N N$ data. We here apply the realization of Ref. [59].

When ChPT is applied to a system of three nucleons, graphs corresponding to 3 NFs appear naturally, in a way fully consistent with the chiral $N N$ interactions. The first-order, including a nonvanishing $3 \mathrm{NF}$ term is N2LO. Until now full calculations, including all graphs of the given order, exist only at N2LO, while at N3LO graphs corresponding to $3 \mathrm{NF}$ have not been incorporated yet. For more details about construction of the $N N$ potential within ChPT approach at N2LO see Ref. [56].

The method has an exceptional advantage as compared to other approaches: a possibility to estimate uncertainties of the obtained predictions. The calculations are performed with a few combinations of the two cut-off parameters, $[\Lambda, \tilde{\Lambda}]$, relevant for regularization of the obtained potential. The range of predictions corresponding to a reasonable choice of the variation intervals of both cutoffs gives an estimation of uncertainty of the theoretical observable. For details on ChPT and the ranges of the regularization cutoffs see Refs. [58,59] and references therein.

\section{Coupled-channels potential with $\Delta$-isobar excitation}

The two-baryon coupled-channels potential (CCP) CD Bonn $+\Delta$, described in Ref. [60], is based on the realistic CD Bonn potential, but in addition includes excitation of a single nucleon to a $\Delta$ isobar, which is considered an active degree of freedom and treated on the same footing as nucleons. In the energy range below the pion-production threshold, where the $\Delta$-isobar excitation is virtual, it is assumed to be a stable baryon with a real mass of $1232 \mathrm{MeV}$. In addition to the purely nucleonic $N N$ component, CCP has a transition component coupling $N N$ and $N \Delta$ states as well as diagonal component between $N \Delta$ states with direct and exchange parts. CCP has contributions from exchanges of $\pi, \rho, \omega$, and $\sigma$ mesons.

Virtual excitation of the $\Delta$ isobar in the $3 N$ system yields an effective $3 \mathrm{NF}$ but also the so-called two-nucleon dispersion. These two contributions usually compete, therefore the net effects of including the $\Delta$ isobar in the potential are smaller than for approaches with the model 3NFs.

The calculations with this force model are performed by the Lisbon-Hannover group. The Coulomb interaction between protons is included using the method of screening and renormalization in the framework of momentum-space integral equations [61]. At $130 \mathrm{MeV}$ the predicted effects for the breakup reaction are sizable for cross sections in some regions of the phase space [26,61], although much less prominent for the analyzing powers, as it has been already demonstrated for $T_{20}$ [27].

\section{Averaging of the theoretical predictions over the experimental acceptance}

The angular ranges around central pointlike geometry, applied in the data analysis, are wide enough to observe effects of averaging of the analyzing powers within these limits. Therefore, similarly as was done for $T_{20}(\xi)$ in Ref. [27], in 
order to compare the data with the theoretical predictions, the same averaging has to be applied to the calculated values of the observables. For that purpose, for each geometry defined by the central values of angles $\theta_{1}^{c}, \theta_{2}^{c}, \varphi_{12}^{c}$, the analyzing powers and cross-section $\sigma_{0}$ values have been calculated for all 27 combinations of angles $\theta_{1}^{c} \pm \frac{1}{2} \Delta \theta_{1}, \theta_{2}^{c} \pm \frac{1}{2} \Delta \theta_{2}$, and $\varphi_{12}^{c} \pm \frac{1}{2} \Delta \varphi_{12}$ and the central values with the step of $1 \mathrm{MeV}$ in variable $S$. It is important to remember that $S$ is defined individually for each kinematical curve, therefore the same values of $S$ for two different geometries (combination of angles) correspond usually to two different pairs of proton energies $\left(E_{1}, E_{2}\right)$. Therefore, further analysis of results obtained at various angular combinations should be carried out on the $\left(E_{1}, E_{2}\right)$ plane rather than using the variable $S$. Analyzing power values obtained for the given geometry and $S$ were weighted with a product of $\sigma_{0}$ and the solid angle factor and placed on the $E_{2}$ versus $E_{1}$ plane. Then, the data obtained in this way were projected onto the curve corresponding to the relativistic kinematics, calculated for the central geometry $\left(\theta_{1}^{c}, \theta_{2}^{c}, \varphi_{12}^{c}\right)$.

This approach is very similar to the analysis of the experimental data (projection of data points on the kinematical curve), therefore it assures that averaging of the theoretically calculated analyzing powers is equivalent to event integration within the ranges set in defining the kinematical configurations. The applied procedure also projects the results of nonrelativistic calculations onto the relativistic kinematics. In this way they can be directly compared to the $S$ distributions of the data, without the necessity to correct for difference of arc lengths calculated along relativistic and nonrelativistic kinematic curves. It has been checked that, due to rather slow variations of the analyzing powers with polar and azimuthal angles, employing a more dense grid of angles for averaging has no influence on the result.

\section{EXPERIMENTAL RESULTS}

Distributions of the vector and tensor analyzing powers as a function of $S(\mathrm{MeV})$ were obtained for 82 geometrical configurations (grid described in subsection IV C), altogether for about 800 kinematical points for each observable. Since the number of observables and configurations makes the presentation of all the results difficult, only examples of distributions compared to various theoretical predictions will be shown in the following. The full set of results obtained by the free fit method can be found in Ref. [41]. Examples presented here have been chosen to illustrate the most important findings, with the emphasis put on problems in describing the data and differences between the predictions. The whole set of experimental results is, however, compared to the theoretical predictions described in Sec. V and the overall quality of reproducing the data is presented. For that purpose a variable defined in analogy to $\chi^{2}$ per degree of freedom is calculated globally, individually for the kinematical configurations and, in addition, for the data sorted according to the energy $E_{\text {rel }}$ of the relative motion of the two protons. The last way of presenting the results is motivated by the earlier studies of cross sections [25,26], showing the importance of that kinematical variable for understanding of the system dynamics.
The above-mentioned $\chi^{2} / \mathrm{DOF}$ is defined as a sum of squares of differences between the experimental results and the theoretically calculated values (averaged according to the description in subsection VD), divided by a sum of squares of statistical uncertainties and by a number of kinematical points in the data set under consideration. In this approach systematical uncertainties of the data and any uncertainty of the theoretical predictions are neglected. The theoretical calculations using realistic potentials (later called $2 \mathrm{~N}$ ) provide very similar results and are treated as a group: they are presented in figures as bands and, in calculations of $\chi^{2} / \mathrm{DOF}$, an average value of all predictions is taken (corresponding to the middle of the band). Calculations using those $N N$ potentials combined with TM99 3NF (2N+TM99) are treated in an analogous way. Predictions obtained within ChPT have well-defined uncertainties, which in some particular regions are quite significant. They are, however, also neglected in this approach and the mean value of the range of the calculated values is taken in $\chi^{2}$ comparisons. Actually, such mean values can correspond, at various kinematical points, to various combinations of the cut-off parameters. Taking into account all the simplifications, the value of the "so-called $\chi^{2} /$ DOF"

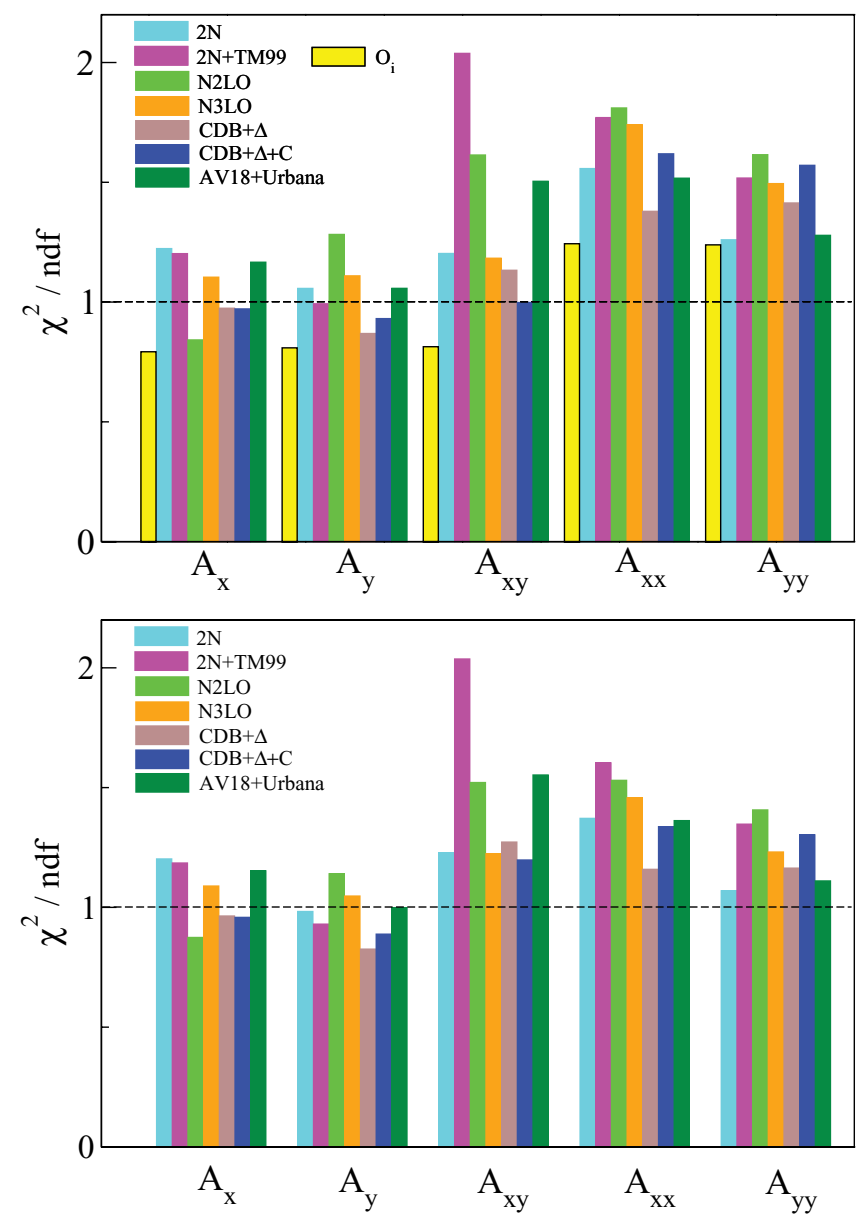

FIG. 7. (Color online) The global $\chi^{2} / \mathrm{DOF}$ results presented as histograms and grouped with respect to the type of observable. Results of the free fit method are shown together with consistency check in the upper panel: $\chi^{2} /$ DOF calculated for $O_{i}$ with respect to 0 . In the lower panel the results of the parity-constrained fit are presented. 
has no precise statistical meaning. However, it can still be used for relative comparison between descriptions provided by different models and, when its value reaches roughly 2 or more, it can be treated as a signal of inconsistency between the model predictions and measured data.

According to the discussion in subsection IV C, the analyzing powers were obtained by the parity-constrained fit, i.e., in the procedure of fitting parity-constrained functions to the linear combinations of asymmetries. For the purpose of a consistency check one exception from this rule took place: the global $\chi^{2} / \mathrm{DOF}$ values obtained in both the free fit method and parity-constrained fit are shown in Fig. 7. The histograms obtained with the free fit method are supplemented with additional bins, corresponding to parityforbidden combinations of observables, defined in Eq. (11). Such combinations, $O_{x}, O_{y}, O_{x x}, O_{x y}$, and $O_{y y}$, were treated similarly to the analyzing power data: their $S$ dependence was obtained for each configuration and $\chi^{2}$ with respect to 0 was calculated. The global $\chi^{2} / \mathrm{DOF}$ values show that all $O_{i}$ are well consistent with 0 . Differences between the results for analyzing powers obtained in the two methods of analysis are small. Generally better agreement between the data and theories (lower $\chi^{2} / \mathrm{DOF}$ ) is reached within the framework of the parity-constrained fit. In the case of $O_{x x}$ and $O_{y y}$ the $\chi^{2} /$ DOF reaches about 1.25 and the difference between the results for $A_{x x}$ and $A_{y y}$, obtained in the two methods of analysis, is the largest, though also not very significant.

On the basis of global $\chi^{2} /$ DOF values it is difficult to point out the theoretical approach leading to the best description of the analyzing power data. All of them seem to be quite successful in reproducing the data, in particular for the vector analyzing powers $A_{x}$ and $A_{y}$, for which all the values of $\chi^{2} / \mathrm{DOF}$ are similar and close to 1 . There are, however, certain hints of problems in the sector of tensor analyzing powers. The values of $\chi^{2} / \mathrm{DOF}$ obtained in the case of $A_{x x}$ are slightly elevated, almost independently of the theoretical approach applied. The predictions of 2N+TM99 provide the least adequate description of the $A_{x y}$ data among all the approaches, but problems are observed also in the case of AV18+Urbana calculations. ChPT calculations at N2LO provide a similar quality of description of this observable as AV18+Urbana; reproducing the data is improved at N3LO. One should remember, however, that here and in what follows, N3LO refers to the calculations based on the N3LO twonucleon forces of Ref. [59] without taking into account the corresponding 3NFs.

Judgment on significance of the effects observed in global $\chi^{2}$ analysis is difficult, therefore the $\chi^{2}$ values have to be examined more locally. On the maps of $\chi^{2} /$ DOF spanned by the studied geometries of the outgoing protons, presented in Fig. 8, one can trace regions of very good data description and regions where departure from that perfect picture occur. White squares represent configurations, for which the particular (parity odd) observable vanishes or for which angular acceptance and collected statistics were too low to perform the fit. Generally, the data description of vector analyzing powers is very good and almost model independent, though one could consider the coupled-channels calculations as the

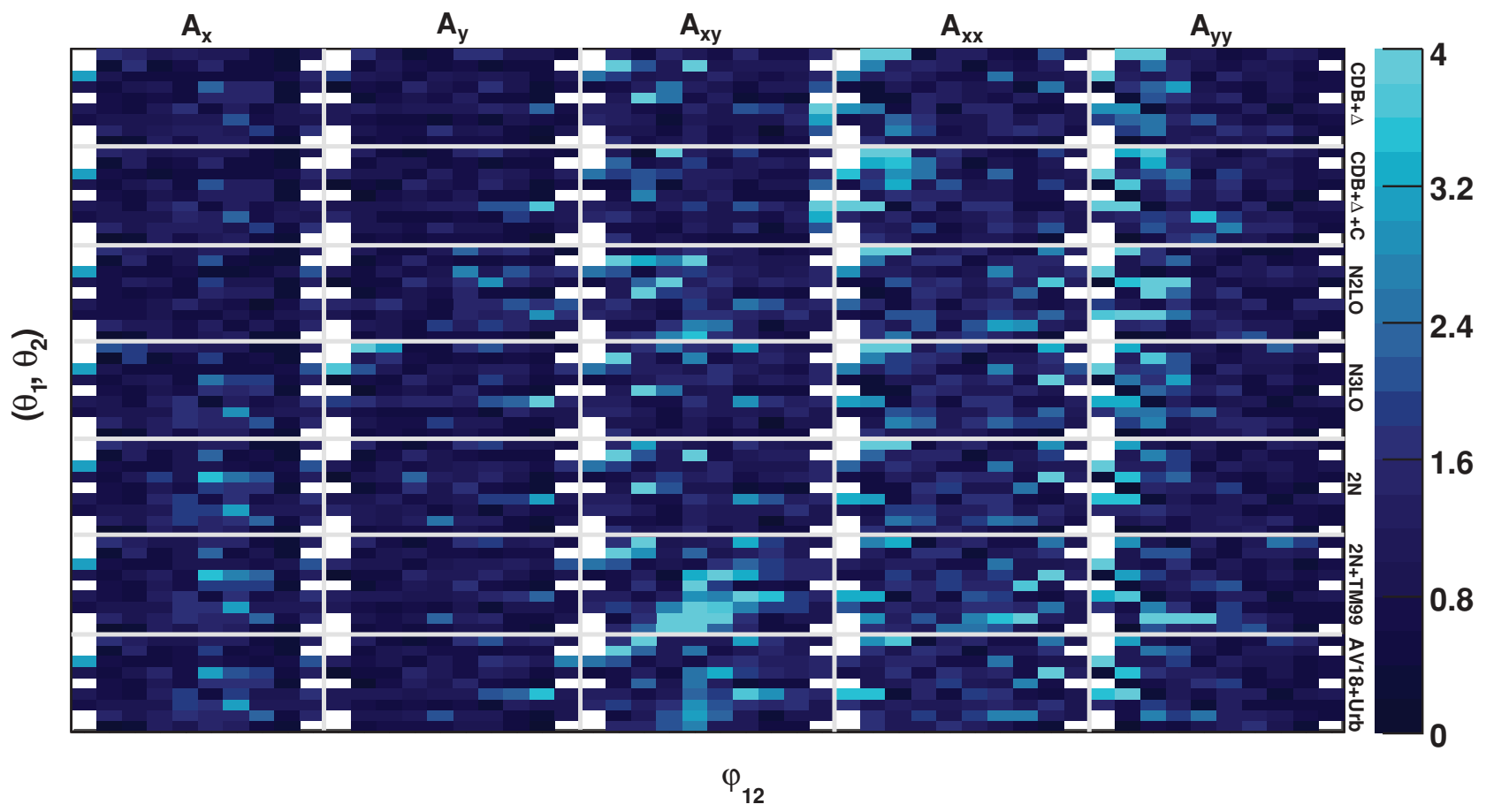

FIG. 8. (Color online) Set of maps of the $\chi^{2} /$ DOF for all studied configurations; columns correspond to the five studied analyzing powers and rows to various theoretical calculations; see labels in the figure. Individual maps are shown for angular configurations defined by the relative azimuthal angle given on the abscissa: from $0^{\circ}$ to $180^{\circ}$ with step of $20^{\circ}$ and by pairs of polar angles $\left(\theta_{1}, \theta_{2}\right)$ on the ordinate, from bottom to top: $\left(15^{\circ}, 15^{\circ}\right),\left(20^{\circ}, 15^{\circ}\right),\left(25^{\circ}, 15^{\circ}\right),\left(30^{\circ}, 15^{\circ}\right),\left(20^{\circ}, 20^{\circ}\right),\left(25^{\circ}, 20^{\circ}\right),\left(30^{\circ}, 20^{\circ}\right),\left(25^{\circ}, 25^{\circ}\right)$ and $\left(30^{\circ}, 25^{\circ}\right)$. White sectors in the map mark the configurations for which the particular observable could not be extracted. 

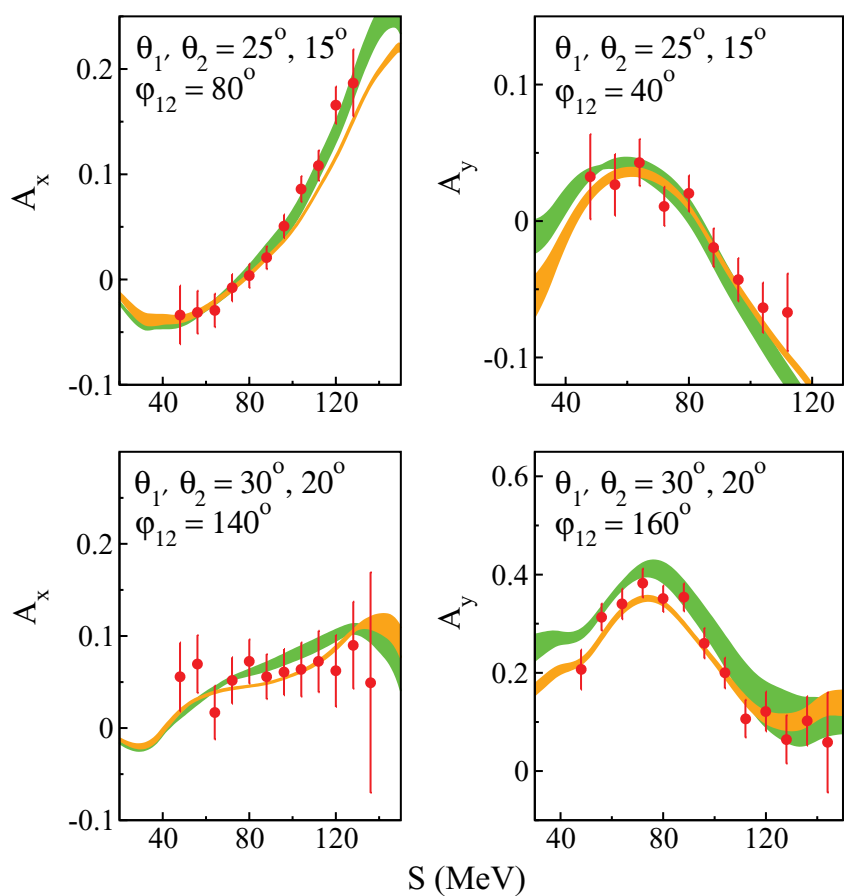

FIG. 9. (Color online) Examples of vector analyzing power distributions obtained for geometries with central values of angles as shown in the panels. Error bars show statistical uncertainties alone. Experimental data are compared to bands representing results of ChPT calculations at N2LO (green, light gray) and N3LO (orange, dark gray).
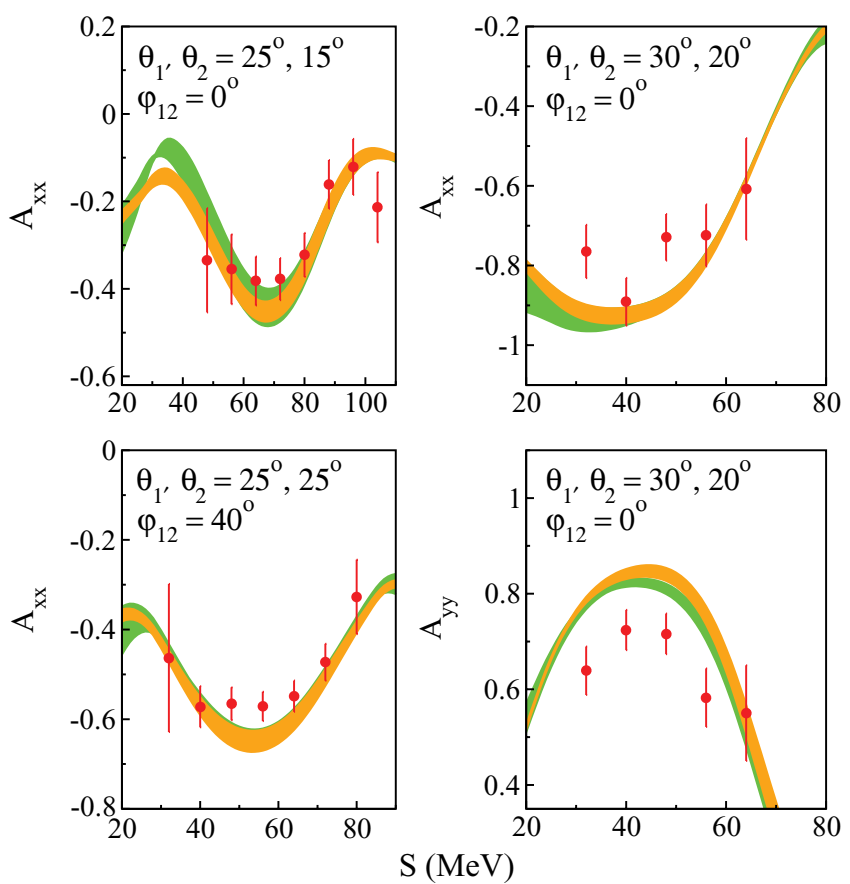

FIG. 11. (Color online) Examples of tensor analyzing power distributions for the geometries where description of the data by theoretical calculations is not perfect. Meaning of the bands as in Fig. 9.
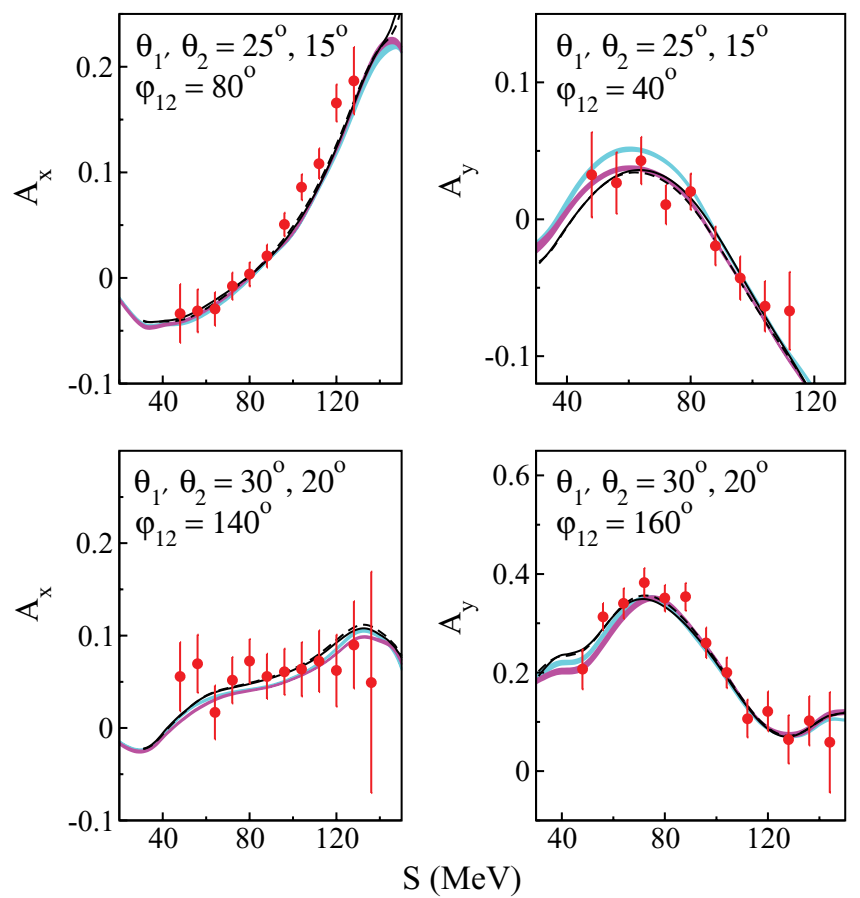

FIG. 10. (Color online) The same data as in Fig. 9 but compared to calculations with realistic potentials without (cyan, light gray) and with (magenta, dark gray) TM99 $3 N$ force. The results of the coupled-channels calculations with the explicit $\Delta$ excitation, with and without Coulomb interaction, are shown as solid and dashed lines, respectively.


FIG. 12. (Color online) The same data as in Fig. 11 but compared to calculations with the realistic potentials. Meaning of lines and bands as in Fig. 10. 

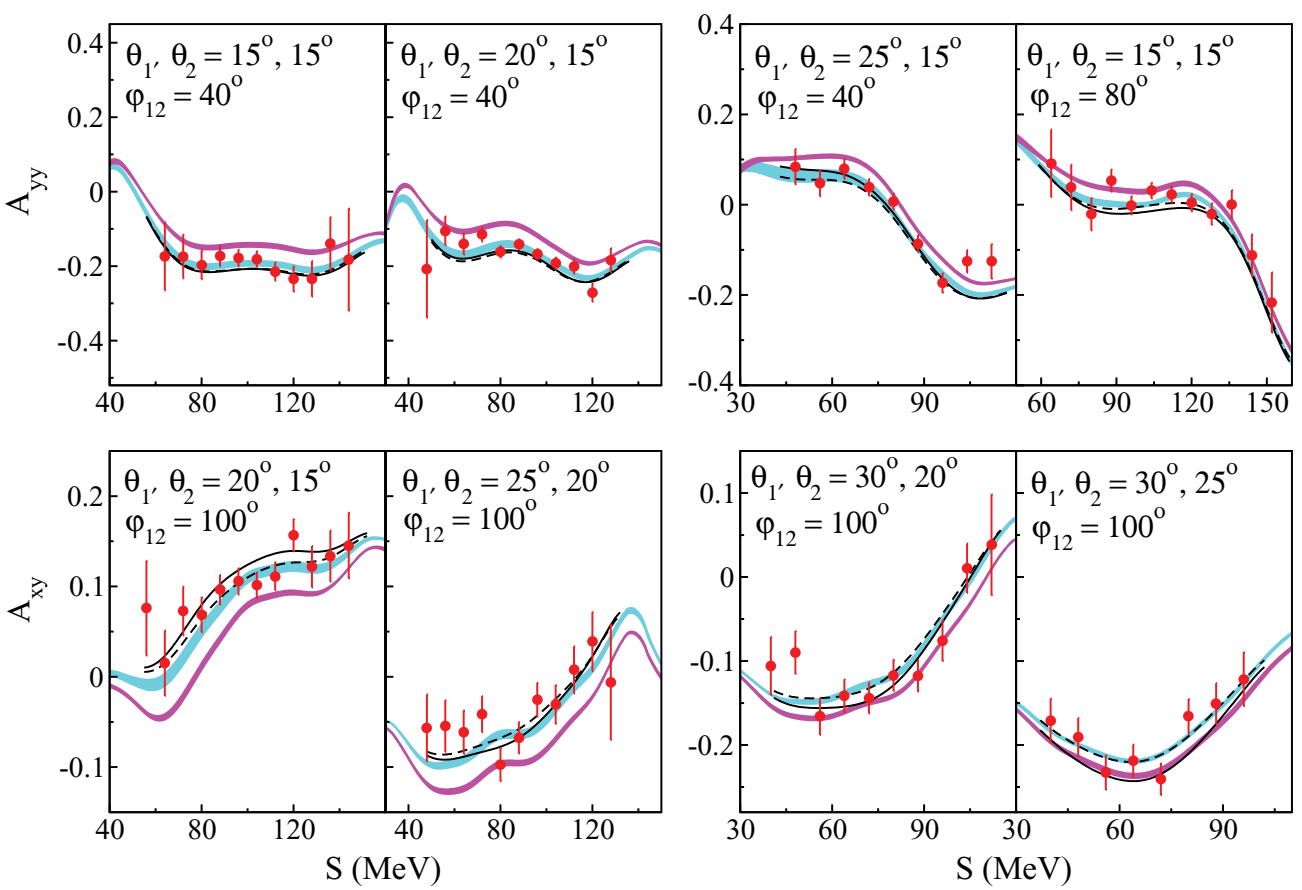

FIG. 13. (Color online) Examples of tensor analyzing power results obtained for configurations in which significant effects of the TM99 $3 \mathrm{NF}$ are predicted. Meaning of lines and bands as in Fig. 10.

most successful in this case. There are certain places at which a slight difference between the results of ChPT at N2LO and N3LO exist. Examples of distributions in function of $S$ obtained for such geometries are shown in Fig. 9. Since calculations with the realistic potentials (with and without 3NF) agree with ChPT predictions at N3LO (cf. Fig. 10), it can be concluded that at N2LO convergence is not reached yet. ChPT calculations at N3LO, though not complete, provide also narrower uncertainty bands than at N2LO.

The analysis of the $\chi^{2} / \mathrm{DOF}$ map for tensor analyzing powers leads to the identification of the regions, which are responsible for the earlier discussed increase of global $\chi^{2} /$ DOF value. Problems with the description of $A_{x x}$ and $A_{y y}$ occur mostly at the lowest $\varphi_{12}$ angles. Examples of such distributions of the data along $S$ curve are shown in Figs. 11 and 12 and compared to various theoretical predictions. The systematic discrepancy between data points and calculated observables is not large but significant: roughly of about 0.1 . It occurs at the same kinematical region, where the simplified analysis lead to identifying problems with the description of $\operatorname{Re} T_{20}$ [27]. This is not a surprise, since these observables are connected by a simple relation: $\operatorname{Re}\left(T_{20}\right)=-\frac{1}{\sqrt{2}}\left(A_{x x}+A_{y y}\right)$. In the right columns of Figs. 11 and 12, $A_{x x}$ and $A_{y y}$ for the same angular configuration are shown, which can be combined together to form $\operatorname{Re} T_{20}$ distribution.

In two of the examples presented in Fig. 12 certain sensitivity of the theoretical predictions to the Coulomb interaction can be observed; however, the description of the data becomes even worse when this important part of interaction is added. In order to compensate for that departure a substantial (and acting in the correct direction) effect of $3 \mathrm{NF}$ would be required.

The maps obtained for $A_{x y}$ confirm the strongest sensitivity of this observable to differences in assumed dynamics. Two types of calculations with the realistic potentials combined with model 3NF, i.e., 2N+TM99 and AV18+Urbana, show similar pattern of inconsistencies with the data, though the effect is much stronger in the case of 2N+TM99. We present in Fig. 13 a set of distributions of the tensor analyzing powers,



FIG. 14. Quality of description of vector analyzing powers given by various models, presented as $\chi^{2}$ per degree of freedom in function of the relative energy of the two breakup protons. 


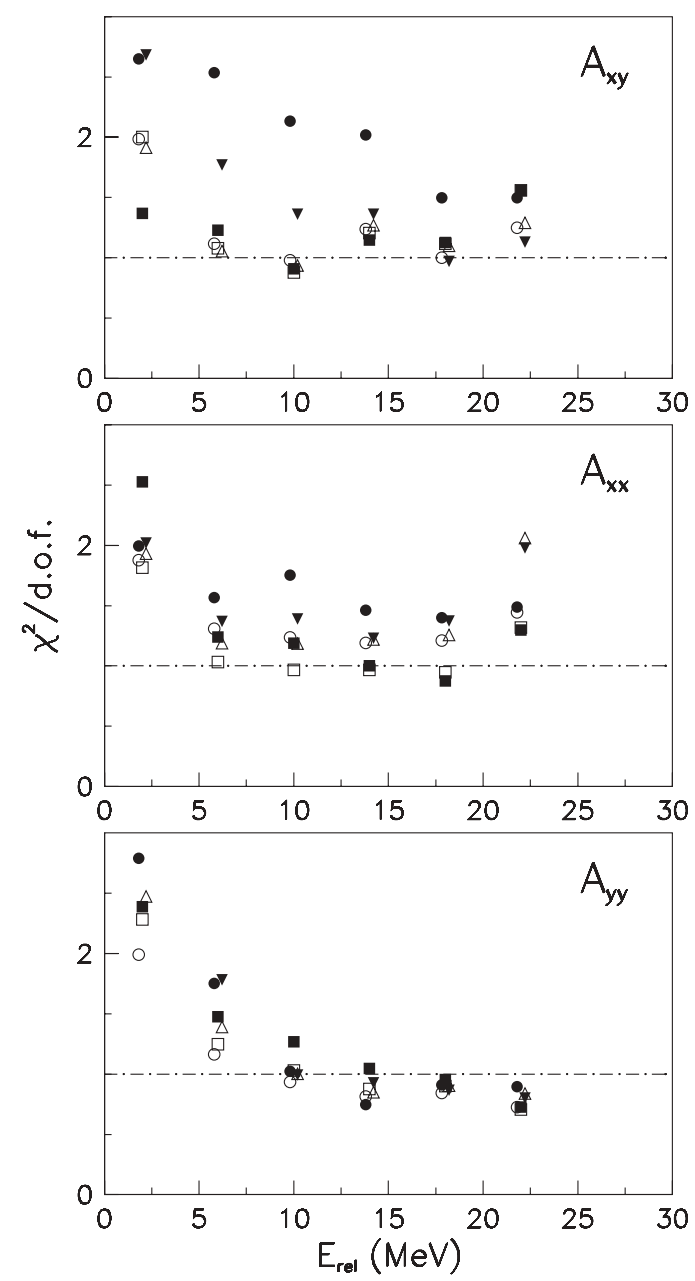

FIG. 15. The same as in Fig. 14 but for tensor analyzing powers.

where the TM99 3NF effect is significant. Among them there are configurations in which inclusion of the TM99 3NF improves the description. Alas, in several other configurations the calculations including the $3 \mathrm{NF}$ contributions lead to a worse agreement with the experimental data. Coulomb effects predicted for $A_{x y}$ are rather small, but in some regions their magnitude is non-negligible and they can be comparable to the effects of the TM99 3NF (e.g., for configuration $\theta_{1}=30^{\circ}$, $\theta_{2}=25^{\circ}, \varphi_{12}=100^{\circ}$, as shown in Fig. 13).

The energy of the relative motion of the two outgoing protons is an important kinematical variable in pointing out various effects in the cross sections, as presented in Refs. [25,26]. Therefore dependence of $\chi^{2} / \mathrm{DOF}$ on $E_{\text {rel }}$ has also been studied for the analyzing powers. Figure 14 presents such dependencies obtained for vector analyzing powers. No particular tendency can be observed and a good description of the data by all theories is confirmed. The influences of $3 \mathrm{NFs}$ and Coulomb interactions on vector analyzing powers are practically negligible in the whole studied region. A similar $\chi^{2}$ analysis for the tensor analyzing powers is shown in Fig. 15. $A_{x y}$ is well described by purely $N N$ interactions, while inclusion of TM99 3NF worsens the agreement, practically in the whole range of relative energies with exception of the highest one. In the case of $A_{x x}$ and $A_{y y}$ the largest discrepancy between the data and all calculations is present for the lowest $E_{\text {rel }}$. The problem with description of $A_{x x}$ at low energies is even increased when Coulomb interactions are included in the calculations.

\section{SUMMARY AND OUTLOOK}

Vector and tensor analyzing powers have been obtained for 82 kinematical configurations of the ${ }^{1} \mathrm{H}(\vec{d}, p p) n$ breakup reaction, covering a significant part of the phase space. The experimental results have been compared to various theoretical calculations. They comprise predictions based on the realistic $N N$ (CD Bonn, AV18, Nijm I, and Nijm II) potentials alone and combined with the TM99 and Urbana $3 \mathrm{NF}$ models. Moreover, the data are confronted with the results of the coupled-channels approach based on the CD-Bonn $+\Delta$ potential, with or without Coulomb interactions included. Finally, the results are compared to the observables obtained within the ChPT framework at N2LO including full dynamics and, currently not complete, calculations at N3LO.

In the majority of the studied configurations and observables all the theoretical predictions agree with each other and describe the data very well. In particular this is true for vector analyzing powers in the whole studied region of the breakup phase space. The situation is more complicated for the tensor analyzing powers, for which, in spite of a general success of the theoretical description, certain discrepancies are observed. For $A_{x y}$ such discrepancies usually appear or are enhanced when the $3 N$ forces, TM99 or Urbana, are included. On the other hand, problems with describing $A_{x x}$ and $A_{y y}$ are limited to the lowest relative energies and are present for all theoretical approaches. Effects of Coulomb interactions are small and distributed rather chaotic in the studied part of the phase space.

Description of the data within the ChPT framework is of very similar quality to that of the other approaches. There are, however, hints that calculations at N3LO are necessary to obtain precise results at this energy, therefore complete calculations (including $3 \mathrm{NF}$ contributions) at that order would be of high importance. One can also expect an improvement of data description when calculations with explicit $\Delta$ degrees of freedom in ChPT [62] become available for the breakup reaction. This approach to ChPT leads to a better convergence of the results and to a reduction of the theoretical uncertainties.

Generally, tensor analyzing powers are more sensitive to details of dynamics, like effects of $3 \mathrm{NF}$ or Coulomb interaction, than vector analyzing powers, what coincides with the observations inferred from the elastic scattering at the same energy [17]. In contrast to the data presented here, the measurements of the breakup reaction at higher energies also hint at problems with a proper description of the vector analyzing powers [28-31]. The observed discrepancies seem to be stronger in proton analyzing powers than in the deuteron ones.

The overall picture drawn here shows that even though in general all presented analyzing powers are quite well reproduced by modern theoretical approaches, there remain regions of unexplained discrepancies, pointing at still persisting flaws in the $3 \mathrm{NF}$ models, in particular in their spin structure. This conclusion is supported by data for various polarization observables at higher energies [28-31]. 


\section{ACKNOWLEDGMENTS}

This work was partially supported by the Polish 2008-2010 science founds as a research Project No. N N202 174635 and by the Polish 2009-2011 science founds as a research Project No. N N202 034836. It is also a part of the research program of the "Stichting voor Fundamenteel Onderzoek der Materie" (FOM) with financial support from the "Nederlandse Organisatie voor Wetenschappelijk Onderzoek" (NWO). The numerical calculations were performed on the supercomputer cluster of JIC in Jülich, Germany.
[1] A. Nogga, H. Kamada, and W. Glöckle, Phys. Rev. Lett. 85, 944 (2000).

[2] M. Viviani, Nucl. Phys. A 631, 111c (1998).

[3] S. C. Pieper and R. B. Wiringa, Annu. Rev. Nucl. Part. Sci. 51, 53 (2001).

[4] P. Navrátil, V. G. Gueorguiev, J. P. Vary, W. E. Ormand, and A. Nogga, Phys. Rev. Lett. 99, 042501 (2007).

[5] H. Witala, W. Glöckle, D. Hüber, J. Golak, and H. Kamada, Phys. Rev. Lett. 81, 1183 (1998).

[6] K. Hatanaka et al., Phys. Rev. C 66, 044002 (2002).

[7] P. Mermod et al., Phys. Lett. B 597, 243 (2004).

[8] A. Ramazani-Moghaddam-Arani et al., Phys. Rev. C 78, 014006 (2008).

[9] E. J. Stephenson, H. Witała, W. Glöckle, H. Kamada, and A. Nogga, Phys. Rev. C 60, 061001(R) (1999).

[10] R. Bieber et al., Phys. Rev. Lett. 84, 606 (2000).

[11] H. Sakai et al., Phys. Rev. Lett. 84, 5288 (2000).

[12] K. Sekiguchi et al., Phys. Rev. C 70, 014001 (2004).

[13] K. Ermisch et al., Phys. Rev. Lett. 86, 5862 (2001); Phys. Rev. C 71, 064004 (2005)

[14] B. v. Przewoski et al., Phys. Rev. C 74, 064003 (2006).

[15] H. R. Amir-Ahmadi et al., Phys. Rev. C 75, 041001(R) (2007).

[16] H. Mardanpour et al., Eur. Phys. J. 31, 383 (2007).

[17] E. Stephan et al., Phys. Rev. C 76, 057001 (2007).

[18] St. Kistryn et al., Nucl. Phys. A 548, 49 (1992); J. Balewski et al., ibid. 581, 131 (1995).

[19] M. Allet et al., Phys. Rev. C 50, 602 (1994); Phys. Lett. B 376, 255 (1996).

[20] J. Zejma et al., Phys. Rev. C 55, 42 (1997).

[21] K. Bodek et al., Few-Body Syst. 30, 65 (2001).

[22] W. Pairsuwan et al., Phys. Rev. C 52, 2552 (1995).

[23] E. Epelbaum, A. Nogga, W. Glockle, H. Kamada, Ulf-G. Meissner, and H. Witala, Phys. Rev. C 66, 064001 (2002).

[24] J. Kuroś-Żołnierczuk, H. Witala, J. Golak, H. Kamada, A. Nogga, R. Skibinski, and W. Glockle, Phys. Rev. C 66, 024004 (2002).

[25] St. Kistryn et al., Phys. Rev. C 68, 054004 (2003); 72, 044006 (2005).

[26] St. Kistryn et al., Phys. Lett. B 641, 23 (2006).

[27] E. Stephan et al., Eur. Phys. J. A 42, 13 (2009).

[28] M. Eslami-Kalantari et al., Mod. Phys. Lett. A 24, 839 (2009).

[29] H. Mardanpour et al., Phys. Lett. B 687, 149 (2010).

[30] H. O. Meyer et al., Phys. Rev. Lett. 93, 112502 (2004).

[31] K. Sekiguchi et al., Phys. Rev. C 79, 054008 (2009).

[32] G. G. Ohlsen, R. E. Brown, F. D. Correll, and R. A. Hardekopf, Nucl. Instrum. Methods 179, 283 (1981).

[33] H. R. Kremers and A. G. Drentje, in Polarized Gas Targets and Polarized Beams, AIP Conf. Proc. No. 421 (AIP Woodbury, NY, 1997), p. 507.
[34] N. Kalantar-Nayestanaki, J. Mulder, and J. Zijlstra, Nucl. Instrum. Methods A 417, 215 (1998).

[35] N. Kalantar-Nayestanaki et al., Nucl. Instrum. Methods A 444, 591 (2000).

[36] S. Schadmand et al., Nucl. Instrum. Methods A 423, 174 (1999).

[37] St. Kistryn, C. P. Bee, and P. Eberhardt, Proceedings of the Sixth International Conference on Electronics for Particle Physics, LeCroy Corporation, Chestnut Ridge New York, 28-29 May 1997, edited by G. J. Blanar and R. L. Sumner.

[38] H. G. Essel and N. Kurz, IEEE Trans. Nucl. Sci. 47, 337 (2000).

[39] J. B. Birks, Proc. Phys. Soc. A 64, 874 (1951).

[40] C. N. Chou, Phys. Rev. 87, 904 (1952).

[41] R. Sworst, Ph.D. thesis, Jagiellonian University, 2009.

[42] K. Suda et al., Nucl. Instrum. Methods A 572, 745 (2007).

[43] R. Machleidt, F. Sammarruca, and Y. Song, Phys. Rev. C 53, R1483 (1996); R. Machleidt, ibid. 63, 024001 (2001).

[44] R. B. Wiringa, V. G. J. Stoks, and R. Schiavilla, Phys. Rev. C 51, 38 (1995).

[45] V. G. J. Stoks, R. A. M. Klomp, C. P. F. Terheggen, and J. J. deSwart, Phys. Rev. C 49, 2950 (1994).

[46] J. Fujita and H. Miyazawa, Prog. Theor. Phys. 17, 360 (1957).

[47] S. A. Coon and W. Glöckle, Phys. Rev. C 23, 1790 (1981).

[48] A. Nogga, D. Hüber, H. Kamada, and W. Glöckle, Phys. Lett. B 409, 19 (1997).

[49] S. A. Coon and H. K. Han, Few-Body Syst. 30, 131 (2001).

[50] B. S. Pudliner, V. R. Pandharipande, J. Carlson, S. C. Pieper, and R. B. Wiringa, Phys. Rev. C 56, 1720 (1997).

[51] W. Glöckle et al., Phys. Rep. 274, 107 (1996), and references therein.

[52] D. Hueber, H. Kamada, H. Witala, and W. Gloeckle, Acta Phys. Pol. B 28, 1677 (1997).

[53] H. Witała, J. Golak, and R. Skibiński, Phys. Lett. B 634, 374 (2006); R. Skibiński, H. Witała, and J. Golak, Eur. Phys. J. A 30, 369 (2006).

[54] A. Deltuva, Phys. Rev. C 80, 064002 (2009).

[55] U. van Kolck, Nucl. Phys. A 680, 17c (2001).

[56] E. Epelbaum, H.-W. Hammer, and U.-G. Meiner, Rev. Mod. Phys. 81, 1773 (2009).

[57] S. Weinberg, Phys. Lett. B 251, 288 (1990); Nucl. Phys. B 363, 3 (1991).

[58] E. Epelbaum, Nucl. Phys. A 737, 43 (2004).

[59] E. Epelbaum, W. Glöckle, and U.-G. Meißner, Nucl. Phys. A 747, 362 (2005).

[60] A. Deltuva, K. Chmielewski, and P. U. Sauer, Phys. Rev. C 67, 034001 (2003); A. Deltuva, R. Machleidt, and P. U. Sauer, ibid. 68, 024005 (2003).

[61] A. Deltuva, A. C. Fonseca, and P. U. Sauer, Phys. Rev. C 72, 054004 (2005); 73, 057001 (2006).

[62] H. Krebs, in Proceedings of the Sixth International Workshop on Chiral Dynamics, CD09, PoS(CD09)024. 